\title{
Elimination of $\beta$-Thioalkoxy Alcohols under Mitsunobu Conditions. A New Synthesis of Conjugated Enynes from Propargylic Dithioacetals
}

\author{
Chih-Wei Chen and Tien-Yau Luh* \\ Department of Chemistry, National Taiwan University, Taipei, Taiwan 106, Republic of China
}

tyluh@ntu.edu.tw

Received July 3, 2008

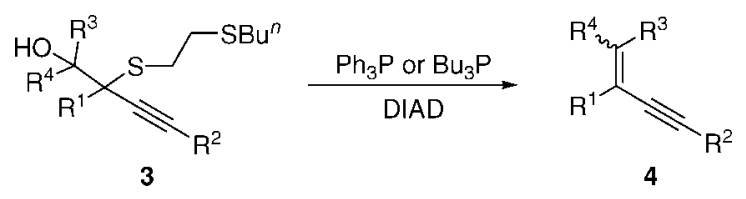

Treatment of propargylic dithiolanes 1 with ${ }^{n} \mathrm{BuLi}$ followed by a carbonyl electrophile yields the corresponding homopropargylic alcohol 3. Upon treatment with 2 equiv of $\mathrm{PPh}_{3}$ and DIAD, elimination of SR and $\mathrm{OH}$ moieties from $\mathbf{3}$ affords the corresponding olefins $\mathbf{4}$ in moderate to good yield. The reaction can be considered an alternative of McMurry coupling of two different carbonyl equivalents.

\section{Introduction}

Carbon-carbon double bond formation by means of elimination of $\beta$-heteroatom substituted alcohols is well-documented. ${ }^{1-6}$ The starting material can be easily accessible from a heteroatomstabilized carbanion and a carbonyl electrophile. We recently reported that aryl- or alkynyl-substituted $\beta$-hydroxythioethers $\mathbf{3}$, obtained from the reaction of dithioacetals $\mathbf{1}$ with BuLi and then with an aldehyde $\mathbf{2}$, can readily undergo low-valent ironpromoted elimination reaction leading to the stereoselective synthesis of olefins 4 (eq 1).,8 The net reaction can be considered as an alternative of McMurry coupling of two different carbonyl equivalents. It is known that oxiranes, ${ }^{9}$ aziridines, ${ }^{10}$ as well as thiiranes ${ }^{11}$ are obtained selectively from

(1) (a) Smith, M. B.; March, J. March's Advanced Organic Chemistry: Reactions, Mechanisms and Structure, 6th ed.; Wiley: Hoboken, NJ, 2007; pp 1534-1546. (b) Bruckner, R. Advanced Organic Chemistry: Reaction Mechanisms; Harcourt: San Diego, CA, 2002; pp 160-167. (c) Concellón, J. M.; Rodríguez-Solla, H. Chem. Soc. Rev. 2004, 33, 599. (d) Shinokubo, H.; Oshima, K. Synlett 2000, 322.

(2) Corey, E. J.; Winter, A. E. J. Am. Chem. Soc. 1963, 85, 2677.

(3) For a review, see: Wong, H. N. C.; Fok, C. C. M.; Wong, T. Heterocycles 1987, 26, 1345.

(4) For reviews, see: (a) Maercker, A. Org. React. 1965, 14, 270. (b) Wadsworth, W. S. Org. React. 1977, 25, 73. (c) Maryanoff, B. E.; Reitz, A. B. Chem. Rev. 1989, 89, 863. (d) Shen, Y.-C. Acta Chim. Sin. 2000, 58, 253.

(5) For reviews, see: (a) Peterson, D. J. J. Org. Chem. 1968, 33, 780. (b) Ager, D. J. Org. React. 1990, 38, 1. (c) Barrett, A. G. M.; Hill, J. M.; Wallace, E. M.; Flygare, J. A. Synlett 1991, 764. (d) van Staden, L. F.; Gravestock, D.; Ager, D. J. Chem. Soc. Rev. 2002, 31, 195.

(6) (a) Kocienski, P. J. In Comprehensive Organic Synthesis; Trost, B. M Fleming, I., Eds.; Pergamon: Oxford, UK, 1991; Vol. 6 (Winterfeldt, E., Ed.), pp 975-1039. (b) Blakemore, P. R. J. Chem. Soc., Perkin Trans. 1 2002, 2563.

(7) Huang, L.-F.; Chen, C. -W.; Luh, T. Y. Org. Lett. 2007, 9, 3663.

(8) A related coupling of a propargylic dithioacetal with a carbonyl compound in the presence of $\mathrm{Cp}_{2} \mathrm{Ti}\left[\mathrm{P}(\mathrm{OEt})_{3}\right]_{2}$ has recently been disclosed. See: Takeda, T.; Ozaki, M.; Kuroi, S.; Tsubouchi, A. J. Org. Chem. 2005, 70, 4233. the corresponding $\beta$-heteroatom-substituted alcohols. In addition, the sulfur moiety of an episulfonium ion can be extruded in the presence of a nucleophile (e.g., $\mathrm{Bu}_{3} \mathrm{P}$ or $\mathrm{Et}_{3} \mathrm{~N}$ ) to give alkenes (eq 2). ${ }^{12}$ It is envisaged that the hydroxyl group in $\mathbf{3}$ may be converted into a suitable leaving group so that intramolecular displacement may occur to generate in situ an episulfonium ion 5. A similar extrusion process may lead to the corresponding alkenes. In this paper, we report an unprecedented protocol for the elimination of $\beta$-hydroxythioethers under Mitsunobu condi-

(9) (a) Ferrier, R. J.; Schmidt, P.; Tyler, P. C. J. Chem. Soc., Perkin Trans. 1 1985, 2, 301. (b) Callam, C. S.; Gadikota, R. R.; Lowary, T. D. J. Org. Chem. 2001, 66, 4549. (c) Dave, R.; Sasaki, N. A. Org. Lett. 2004, 6, 15. (d) Koyama, Y.; Lear, M. J.; Yoshimura, F.; Ohashi, I.; Mashimo, T.; Hirama, M. Org. Lett. 2005, 7, 267. (e) Thoret, S.; Gueritte, F.; Guenard, D.; Dubois, J. Org. Lett. 2006, 8, 2301. (f) Bai, Y.; Lowary, T. L. J. Org. Chem. 2006, 71, 9672. (g) Garcia-Delgado, N.; Riera, A.; Verdaguer, X. Org. Lett. 2007, 9, 635.

(10) (a) Bose, A. K.; Sahu, D. P.; Manhas, M. S. J. Org. Chem. 1981, 46, 1229. (b) Ibuka, T.; Mimura, N.; Aoyama, H.; Akaji, M.; Ohno, H.; Miwa, Y.; Taga, T.; Nakai, K.; Tamamura, H.; Fujii, N.; Yamamoto, Y. J. Org. Chem. 1997, 62, 999. (c) Hillier, M. C.; Davidson, J. P.; Martin, S. F. J. Org. Chem. 2001, 66, 1657. (d) Ohno, H.; Miyamura, K.; Tanaka, T.; Oishi, S.; Toda, A.; Takemoto, Y.; Fujii, N.; Ibuka, T. J. Org. Chem. 2002, 67, 1359. (e) Galonic, D. P.; Ide, N. D.; van der Donk, W. A.; Gin, D. Y. J. Am. Chem. Soc. 2005, 127, 7359. (f) Gandhi, S.; Bisai, A.; Prasad, B. A. B.; Singh, V. K. J. Org. Chem. 2007, 72, 2133. (g) Caldwell, J. J.; Craig, D. Angew. Chem., Int. Ed. 2007, 46, 2631.

(11) Camp, D.; Jenkins, I. D. Aust. J. Chem. 1990, 43, 161.

(12) (a) Gybin, A. S.; Smit, A. S. Tetrahedron 1980, 36, 1361. (b) Denis, J. N.; Dumont, W.; Krief, A. Tetrahedron Lett. 1979, 42, 4111. (c) Helmkamp, G. K.; Pettitt, D. J. J. Org. Chem. 1960, 25, 1754. (d) Mukaiyama, T.; Shiono, M.; Sato, T. Chem. Lett. 1974, 1, 37. (e) Mukaiyama, T.; Shiono, M.; Watanabe, K.; Onaka, M. Chem. Lett. 1975, 7, 871. (f) Mukaiyama, T.; Imaoka, M. Chem. Lett. 1978, 4, 413.

(13) For reviews on Mitsunobu reaction, see: (a) Mitsunobu, O. Synthesis 1981, 1. (b) Hughes, D. L. OrgReact. (N.Y.) 1992, 42, 335. (c) But, T. Y. S.; Toy, P. H. Chem. Asian J. 2007, 2, 1340. 
TABLE 1. Elimination of $\beta$-Thioalkoxy Alcohols under Mitsunobu Conditions

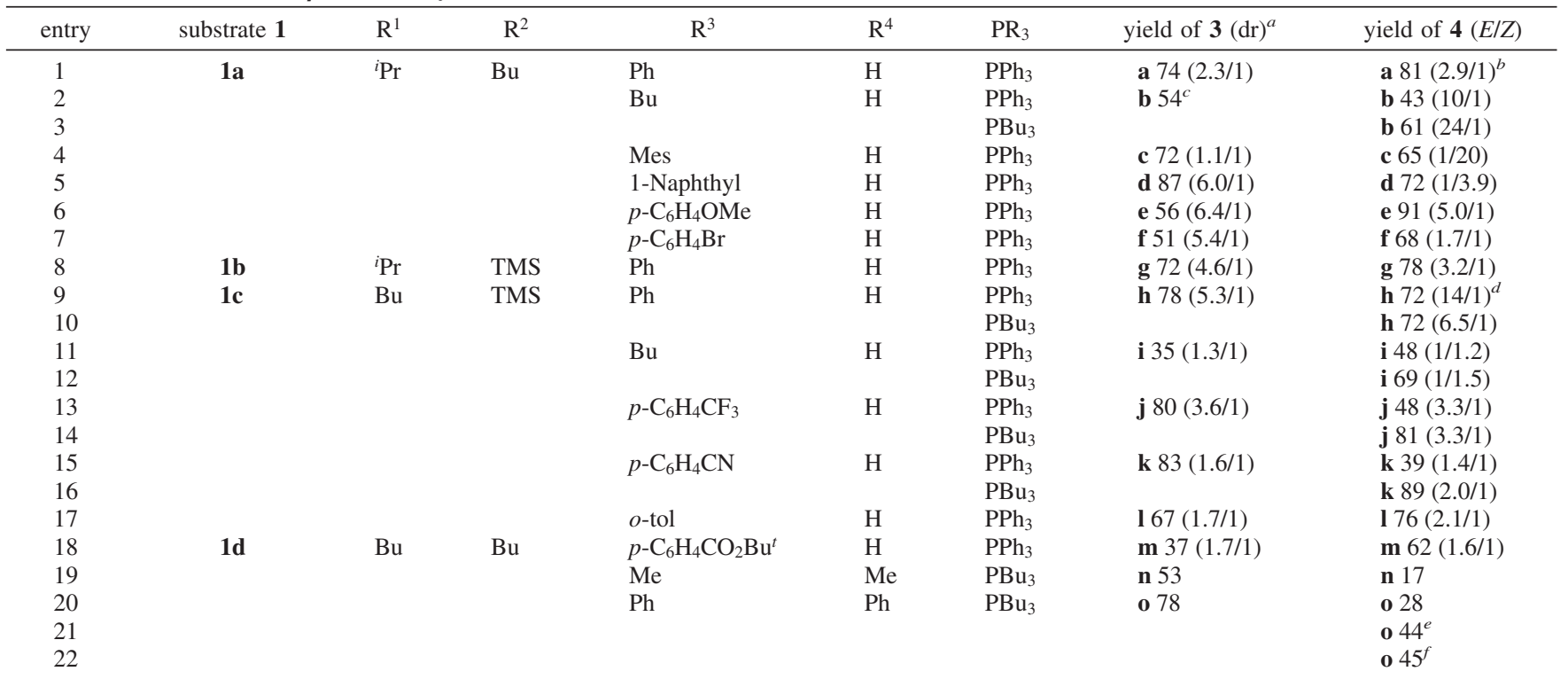

${ }^{a}$ Diastereomeric ratio (major isomer/minor isomer) was determined by ${ }^{1} \mathrm{H}$ NMR integrals. ${ }^{b}$ When 1 equiv of Mitsunobu reagent was used, the yield was $63 \%{ }^{c} \mathrm{dr}$ cannot be resolved by ${ }^{1} \mathrm{H}$ NMR. ${ }^{d}$ Identical results were obtained when the reaction was carried out from -78 to $-30{ }^{\circ} \mathrm{C}$. ${ }^{e}$ Reaction time $=48$ h. ${ }^{f} 4$ equiv each of DIAD and $\mathrm{Bu}_{3} \mathrm{P}$ were used.

tions for the olefination of propargylic dithioacetals with a carbonyl compound.

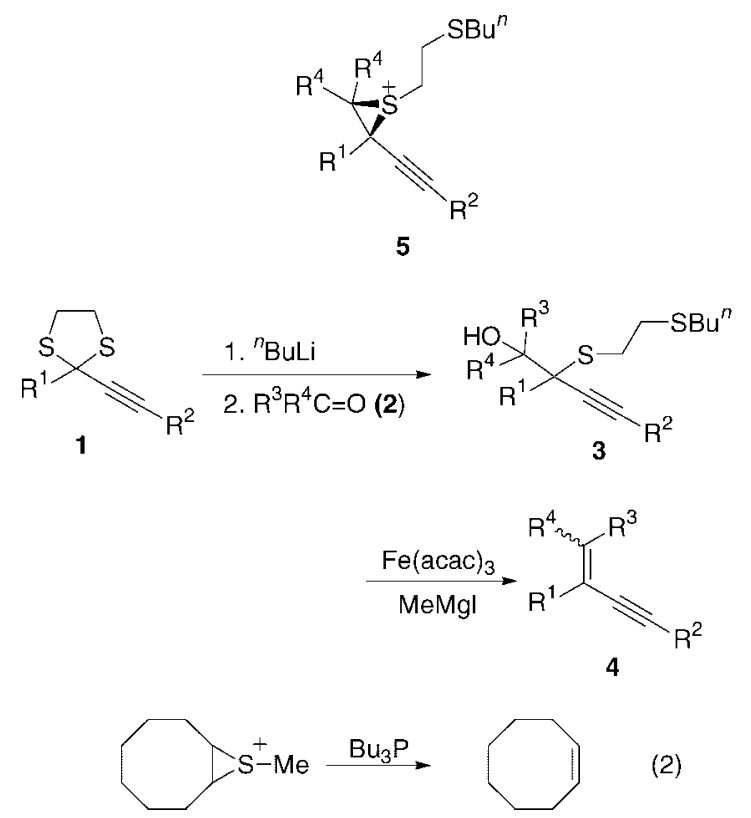

\section{Results and Discussion}

The starting $\mathbf{3}$ were obtained from the reaction of $\mathbf{1}$ with ${ }^{n} \mathrm{BuLi}$ followed by treatment with $\mathbf{2}$ according to literature procedures. ${ }^{7}$ In the beginning of this study, several Lewis acids $\left(\mathrm{BF}_{3} \cdot \mathrm{OEt}_{2}\right.$, $\mathrm{BBr}_{3}$, and $\mathrm{TiCl}_{4}$ ) were employed to react with $\mathbf{3 c}$, only starting material being recovered in $63-85 \%$ yields. Interestingly, when 3 was allowed to react with 2 equiv each of DIAD and $\mathrm{Ph}_{3} \mathrm{P}$ in THF at rt for $18 \mathrm{~h}$ under Mitsunobu conditions, ${ }^{13,14}$ alkene 4

(14) $\alpha$-Hydroxyalkyldithiolanes have been known to be converted into 2-azido-1,4-dithianes under Mitsunobu conditions in the presence of sodium azide. 1,2-Sulfur migration has been suggested. The corresponding 2,3-dihydro1,4- dithiins are isolated as side products. See: Afonso, C. A. M.; Barros, M. T.; Maycock, C. D. Tetrahedron 1999, 55, 801 . was obtained in moderated to good yield. Representative examples are summarized in Table 1. The stereochemical assignments for $\mathbf{4}$ were based on NOE experiments. Mitsunobu reaction of $\mathbf{3 p}$ obtained from 1 e [(1). BuLi, (2) $\mathrm{PhCHO}$, (3) TBSCl, imidizole] under the same conditions gave the corresponding enyne $\mathbf{4 p}(E / Z=1.6 / 1)$ in $38 \%$ yield (eq 3$)$.

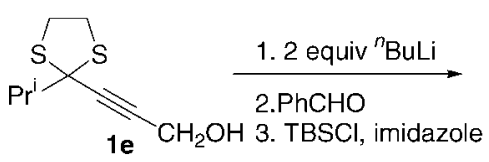

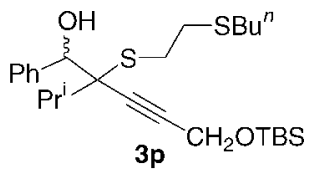

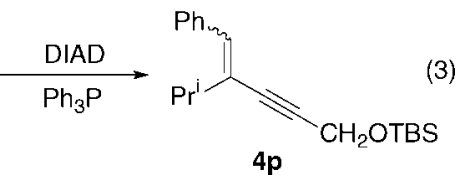

Interestingly, the yield of $\mathbf{4}$ was significantly improved when $\mathrm{Ph}_{3} \mathrm{P}$ was replaced by $\mathrm{Bu}_{3} \mathrm{P}$ (entries $3,12,14$, and 16). Tertiary alcohols are normally quite unreactive under typical Mitsunobu conditions. ${ }^{15}$ Indeed, when $\mathbf{3 n}$ was allowed to react with DIAD and $\mathrm{Ph}_{3} \mathrm{P}$ at $\mathrm{rt}$, no reaction was observed. However, alkenes $\mathbf{4 n}$ and $4 \mathbf{o}$ were isolated in low yield from the corresponding $\mathbf{3 n}$ and 3o when $\mathrm{Bu}_{3} \mathrm{P}$ was employed (entries 19-22), starting materials being recovered in $40-80 \%$ yield. It is noteworthy that longer reaction time (entry 21) or excess of Mitsunobu reagent (entry 22) gave better yield of the reaction.

The reaction can also be carried out in one pot from $\mathbf{1}$. Thus, reaction of 1a with BuLi and then with benzaldehyde gave the corresponding alkoxide, which was directly treated with 2 equiv each of DIAD and $\mathrm{Ph}_{3} \mathrm{P}$ to give $\mathbf{4 a}(E / Z=4.5 / 1)$ in $34 \%$ yield.

(15) (a) Takahashi, M.; Ogasawara, K. Tetrahedron: Asymetry 1997, 8, 3125. (b) Lochead, A.; Galli, F.; Jegham, S.; Nedelec, P.; George, P. Synth. Commun. 1999, 29, 799. (c) Yus, M.; Soler, T.; Foubelo, F. Tetrahedron: Asymmetry 2001, 12, 801. (d) Shi, Y.-J.; Hughes, D. L.; McNamara, J. M. Tetrahedron Lett. 2003, 44, 3609. (e) La Clair, J. J. Angew. Chem., Int. Ed. 2006, 45, 2769. 
SCHEME 1. Possible Mechanism for the Formation of 4

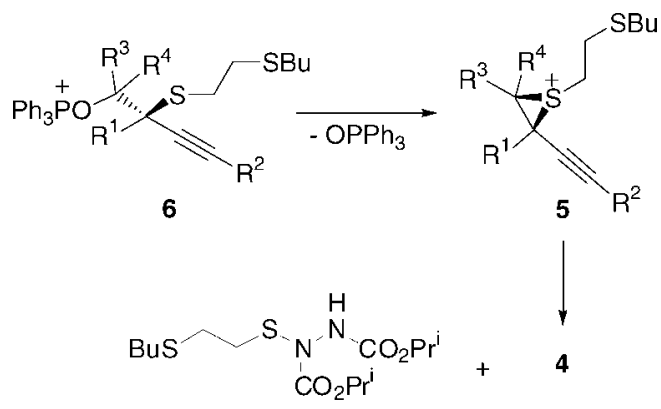

7

It is worthy to mention that the stereoselectivities of the elimination reaction under Mitsunobu conditions were very different from those of the low-valent iron-promoted reactions. ${ }^{7}$ Apparently, the two processes may proceed via very different mechanisms. In addition, the stereoselectivity appeared to be temperature independent (entry 9). It seems plausible that the hydroxyl group in $\mathbf{3}$ would be activated by $\mathrm{R}_{3} \mathrm{P}$ to generate intermediate 6. The phosphine oxide may serve as a leaving group with the concomitant participation of the neighboring sulfur moiety leading to the formation of episulfonium ion $\mathbf{5}$ (Scheme 1). Stereoselective extrusion of the sulfur moiety ${ }^{16}$ may lead to the corresponding olefin stereospecifically. The fate of the sulfur moiety was assigned to be 7 as detected by the highresolution mass spectrometry. ${ }^{16}$ Presumably, the episulfonium ion may be attached by the amidic anion leading to 4 and 7 . These results support the earlier mechanism proposed by Smit et al. ${ }^{12 \mathrm{a}}$

The stereospecificity has been tested by studying the elimination reaction of diasteromerically pure $\beta$-thioalkoxyalcohol. Thus, treatment of $\mathbf{3 k a}$ with 2 equiv each of $\mathrm{Bu}_{3} \mathrm{P}$ and DIAD afforded stereospecifically $Z-4 \mathbf{k}$ in $71 \%$ yield (eq 2 ). On the other hand, the reaction of the other diastereomer $\mathbf{3 k b}(94 \%$ de) under the same conditions afforded $\mathbf{4 k}(E / Z=34 / 1)$ in $71 \%$ yield (eq 5). If the conversion of $\mathbf{6}$ to $\mathbf{5}$ would occur via an $\mathrm{S}_{\mathrm{N}}$ 2-type mechanism, the stereochemistry for $\mathbf{3 k a}$ and $\mathbf{3 k b}$ would thus be assigned.

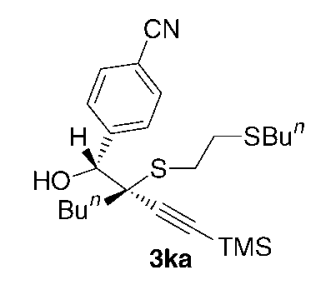<smiles>[Z14]C#C/C(Br)=C\c1ccc(C#N)cc1</smiles><smiles>C[C@H](C#CC#N)[C@@H](O)c1ccc(C#N)cc1</smiles>

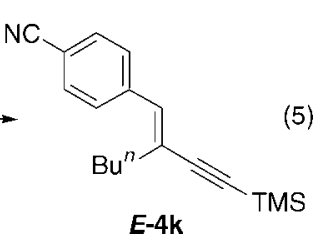

It is interesting to note that a bulky aryl substituent in $\mathbf{3}$ would lead the elimination process to form $Z$ isomer predominantly (entries 4 and 5). Isomerization may take place to give thermodynamically more stable products.

\section{SCHEME 2. Evidence for Thio Migration}<smiles>CCCC(=O)C(CCC)C(C=O)C1=CC=CCC1</smiles><smiles>CCCC(CCC)(c1ccccc1)C(O)(c1cccc2ccccc12)C(C)C</smiles><smiles>C=C(C(CCCC)=C(C)C)c1cccc2ccccc12</smiles>

$11 E / Z=1 / 269 \%$

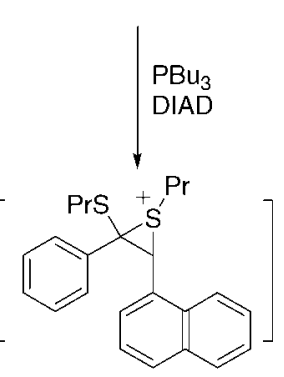

12

To establish the sulfur migration shown in Scheme 1, 8, prepared from the carbanion derived from the corresponding dithioacetal 9 and aldehyde 10, was treated with 2 equiv each of DIAD and $\mathrm{Bu}_{3} \mathrm{P}$ under the usual conditions to give $\mathbf{1 1}(E / Z$ $=1 / 2$ ) in $69 \%$ yield. Apparently, the sulfur migration may take place leading to $\mathbf{1 1}$ via the episulfonium ion intermediate $\mathbf{1 2}$ (Scheme 2). ${ }^{17}$ It is noteworthy that 1,2-thio migration in cyclic dithioacetals is well documented. ${ }^{18}$

When terephthaldehyde was employed, bis-enyne 13 was obtained from in $27 \%$ overall yield from 1a (eq 6). Treatment of 1c with BuLi followed by aldehyde 14 having a dithiolane functionality afforded alcohol $\mathbf{1 5}$ in $45 \%$ yield. Reaction of $\mathbf{1 5}$ under Mitsunobu conditions for $18 \mathrm{~h}$ gave the corresponding endiyne 16 in $68 \%$ yield (eq 7). It is worthy to note that the silyl substituent in $\mathbf{1 6}$ can be further modified by functional group transformation. This strategy could be used for the convergent synthesis of higher homologues of conjugated enynes. A similar approach has been employed toward the synthesis of monodispersed polymers without repetitive units. ${ }^{19}$

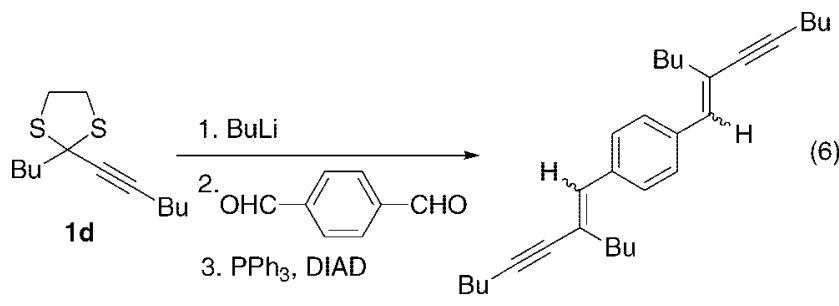

$13 E E / E Z=1 / 3.2$

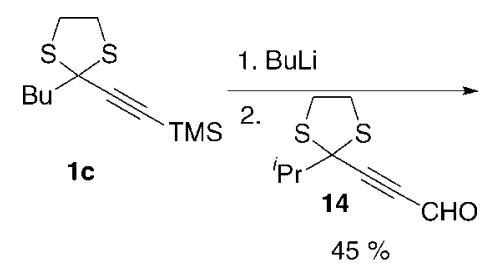<smiles>CCCC1(C#CC(O)CSCC(C)(C)C)SCCS1</smiles>

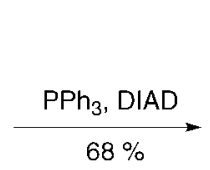<smiles>CC#CC(=CC#CC1(P(C)C)SCCS1)CBr</smiles>

$16 E / Z=1 / 1.4$ 


\section{Conclusions}

In summary, we have demonstrated a new enyne preparation by coupling of a carbonyl compound with a propargylic dithioacetal. A range of substituted enynes can be readily obtained by this protocol. The present reaction can compliment our earlier work ${ }^{7}$ on low-valent iron-promoted elimiation of $\beta$-thioalkoxy alcohols. An episulfonium ion intermediate has been suggested. Both reactions can be considered as an alternative of McMurry-type coupling of two different carbonyl equivalents to give the corresponding olefins.

\section{Experimental Section}

3-(2-Isopropyl-1,3-dithiolan-2-yl)prop-2-yn-1-ol (1e). Under $\mathrm{N}_{2}$, to a THF solution (50 mL) of 2-ethynyl-2-isopropyl-1,3dithiolane $^{7}$ (3.35 g, $20 \mathrm{mmol}, 1.0$ equiv) cooled at $-78^{\circ} \mathrm{C}$ was added dropwise BuLi (10 mL, 1.25 equiv, 2.5 M hexane solution). After stirring $0.5 \mathrm{~h}$, formaldehyde gas was introduced to the mixture until white participate appeared. The mixture was gradually warmed to $\mathrm{rt}$ and stirred for $8 \mathrm{~h}$, quenched with sat. $\mathrm{NH}_{4} \mathrm{Cl}$, washed with brine, and extracted with EtOAc. The organic layer was dried $\left(\mathrm{MgSO}_{4}\right)$ and filtered and the filtrate was evaporated in vacuo to give the residue, which was chromatographed on silica gel (hexane/ EtOAc $=3 / 1)$ to give 1e as a pale yellow liquid $(3.60 \mathrm{~g}, 89 \%) .{ }^{1} \mathrm{H}$ NMR $\left(\mathrm{CDCl}_{3}, 400 \mathrm{MHz}\right) \delta 1.22(\mathrm{~d}, J=6.8 \mathrm{~Hz}, 6 \mathrm{H}), 1.78(\mathrm{t}, J=$ $6.2 \mathrm{~Hz}, 1 \mathrm{H}), 2.27$ (sept, $J=6.7 \mathrm{~Hz}, 1 \mathrm{H}), 3.37-3.60(\mathrm{~m}, 4 \mathrm{H})$, $4.35(\mathrm{~d}, J=6.4 \mathrm{~Hz}, 2 \mathrm{H}) ;{ }^{13} \mathrm{C} \mathrm{NMR}\left(100 \mathrm{MHz}, \mathrm{CDCl}_{3}\right) \delta 21.1$, 39.7, 40.4, 50.9, 66.2, 83.3, 86.4; IR (KBr) •3371 cm-1; HRMS (FAB) (M) calcd for $\mathrm{C}_{9} \mathrm{H}_{15} \mathrm{OS}_{2} 203.0564$, found 203.0566.

General Procedure for Prepartion of Homopropargylic Alcohol 3. Under $\mathrm{N}_{2}$ atmosphere, to a THF solution $(50 \mathrm{~mL})$ of $\mathbf{1}(5.0 \mathrm{mmol}$, 1.0 equi.) cooled at $-78{ }^{\circ} \mathrm{C}$ was added dropwise BuLi $(2.2 \mathrm{~mL}$, 1.10 equiv, $2.5 \mathrm{M}$ hexane solution). After the solution was stirred for $1 \mathrm{~h}$, carbonyl compound (1.0 equiv) in THF $(10 \mathrm{~mL})$ was then added dropwise and the mixture was gradually warmed to $\mathrm{rt}$ and stirred for $2-8 \mathrm{~h}$, quenched with sat. $\mathrm{NH}_{4} \mathrm{Cl}$, washed with brine, and extracted with ether. The organic layer was dried $\left(\mathrm{MgSO}_{4}\right)$ and filtered and the filtrate was evaporated in vacuo to give the residue, which was chromatographed on silica gel (hexane/EtOAc $=10-30 / 1)$ to give 3 .

7-( $\alpha$-Hydroxybenzyl)-7-isopropyl-8,11-dithiapentadec-5-yne (3a): $74 \%(\mathrm{dr}$ ratio $=2.3 / 1)$; major isomer ${ }^{1} \mathrm{H} \mathrm{NMR}\left(\mathrm{CDCl}_{3}, 400 \mathrm{MHz}\right)$ $\delta 0.92(\mathrm{t}, J=6.7 \mathrm{~Hz}, 3 \mathrm{H}), 0.94(\mathrm{t}, J=6.7 \mathrm{~Hz}, 3 \mathrm{H}), 1.12(\mathrm{~d}, J$ $=6.4 \mathrm{~Hz}, 3 \mathrm{H}), 1.34(\mathrm{~d}, J=6.4 \mathrm{~Hz}, 3 \mathrm{H}), 1.34-1.48(\mathrm{~m}, 4 \mathrm{H})$, $1.48-1.60(\mathrm{~m}, 4 \mathrm{H}), 1.88$ (sept, $J=6.4 \mathrm{~Hz}, 1 \mathrm{H}), 2.29$ (t, $J=7.0$ $\mathrm{Hz}, 2 \mathrm{H}), 2.41-2.68(\mathrm{~m}, 6 \mathrm{H}), 3.08(\mathrm{~d}, J=6.8 \mathrm{~Hz}, 1 \mathrm{H}), 4.80(\mathrm{~d}$, $J=6.8 \mathrm{~Hz}, 1 \mathrm{H}), 7.27-7.33(\mathrm{~m}, 3 \mathrm{H}), 7.49-7.58(\mathrm{~m}, 2 \mathrm{H})$;

(17) No extrusion of sulfur moiety to give vinyl sulfide $11^{\prime}$ was observed. The presence of an extra thiolato substituent on olefin in $\mathbf{1 2}$ might result in different behavior.

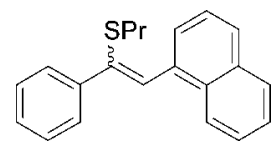

$11^{\prime}$

(18) For examples see: (a) Maldonado, L. A.; Manjarrez, N. Heterocycles 1985, 23, 1985. (b) Caputo, R.; Ferreri, C.; Palumbo, G. Tetrahedron Lett. 1986, 42, 2369. (c) Tani, H.; Inamasu, T.; Tamura, R.; Suzuki, H. Chem. Lett. 1990, 1323. (d) Jekö, J.; Timár, T.; Jaszberenyi, J. C. J. Org. Chem. 1991, 56, 6748. (e) Tani, H.; Kamada, Y.; Azuma, N.; Ono, N. Tetrahedron Lett. 1994, 35, 7051. (f) Kim, J. T.; Kel'in, A. V.; Gevorgyan, V. Angew. Chem., Int. Ed. 2003, 42, 98. (g) Peng, L.; Zhang, X.; Zhang, S.; Wang, J. J. Org. Chem. 2007, 72, 1192. (19) (a) Lee, C.-F.; Yang, L.-M.; Hwu, T.-Y.; Feng, A.-S.; Tseng, J.-C.; Luh, T.-Y. J. Am. Chem. Soc. 2000, 122, 4992. (b) Lee, C.-F.; Lin, C.-Y.; Song, H.-C.; Luo, S.-J.; Tseng, J.-C.; Tso, H.-H.; Luh, T.-Y. Chem. Commun. 2002, 2824. (c) Chou, C.-M.; Chen, W.-Q.; Chen, J.-H.; Lin, C.-L.; Tseng, J.-C.; Lee, C.-F.; Luh, T.-Y. Chem. Asia. J. 2006, 1, 46. (d) For a review, see: Luh, T.-Y.; Lee, C.-F. Eur. J. Org. Chem. 2005, 3839. characteristic ${ }^{1} \mathrm{H}$ NMR signals for the minor isomer: $\delta 2.16$ (sept, $J=6.7 \mathrm{~Hz}), 2.88(\mathrm{~d}, J=4.8 \mathrm{~Hz}, 1 \mathrm{H})$; IR $(\mathrm{KBr}) v 3446 \mathrm{~cm}^{-1}$; HRMS (FAB) $(\mathrm{M}+\mathrm{H})^{+}$calcd for $\mathrm{C}_{19} \mathrm{H}_{29} \mathrm{OS}_{2} 337.1660$, found 337.1666 .

7-(1-Hydroxypentyl)-7-isopropyl-8,11-dithiapentadec-5-yne (3b): $72 \% ;{ }^{1} \mathrm{H}$ NMR $\left(\mathrm{CDCl}_{3}, 400 \mathrm{MHz}\right) \delta 0.92(\mathrm{t}, J=7.4 \mathrm{~Hz}, 9 \mathrm{H})$, $1.02-1.12(\mathrm{~m}, 6 \mathrm{H}), 1.28-1.64(\mathrm{~m}, 14 \mathrm{H}), 1.68-1.80(\mathrm{~m}, 1 \mathrm{H})$, $1.95-2.14(\mathrm{~m}, 1 \mathrm{H}), 2.28$ (t, $J=6.8 \mathrm{~Hz}, 2 \mathrm{H}), 2.50$ (t, $J=7.8 \mathrm{~Hz}$, $2 \mathrm{H}), 2.62-2.98(\mathrm{~m}, 4 \mathrm{H}), 3.56-3.64(\mathrm{~m}, 1 \mathrm{H})$; IR (KBr) $v 3482$ $\mathrm{cm}^{-1}$; HRMS (FAB) $(\mathrm{M}+\mathrm{H})$ calcd for $\mathrm{C}_{21} \mathrm{H}_{41} \mathrm{OS}_{2} 373.2599$, found 373.2599 .

7-( $\alpha$-Hydroxy-2,4,6-trimethylbenzyl)-7-isopropyl-8,11-dithiapentadec-5-yne (3c): $51 \%(\mathrm{dr}$ ratio $=1.1 / 1) ;{ }^{1} \mathrm{H}$ NMR $\left(\mathrm{CDCl}_{3}\right.$, $400 \mathrm{MHz}) \delta 0.82-0.98(\mathrm{~m}, 6 \mathrm{H}), 1.02-1.18(\mathrm{~m}, 6 \mathrm{H}), 1.23-1.48$ $(\mathrm{m}, 6 \mathrm{H}), 1.52-1.66(\mathrm{~m}, 2 \mathrm{H}), 1.70-1.98(\mathrm{~m}, 1 \mathrm{H}), 2.07-2.17(\mathrm{~m}$ $1 \mathrm{H}), 2.23-2.47(\mathrm{~m}, 11 \mathrm{H}), 2.50-2.62(\mathrm{~m}, 2 \mathrm{H}), 2.6-2.84(\mathrm{~m}, 4$ $\mathrm{H}), 5.63-5.68(\mathrm{~m}, 1 \mathrm{H}), 6.81(\mathrm{~s}, 2 \mathrm{H})$; characteristic ${ }^{1} \mathrm{H}$ NMR signals for the major isomer $\delta 2.09(\mathrm{~d}, J=3.6 \mathrm{~Hz}, 1 \mathrm{H}), 5.66(\mathrm{~d}$, $J=3.6 \mathrm{~Hz}, 1 \mathrm{H})$; minor isomer ${ }^{1} \mathrm{H} \mathrm{NMR}\left(\mathrm{CDCl}_{3}, 400 \mathrm{MHz}\right) \delta$ $2.15(\mathrm{~d}, J=3.6 \mathrm{~Hz}, 1 \mathrm{H}), 5.64(\mathrm{~d}, J=3.6 \mathrm{~Hz}, 1 \mathrm{H})$; IR $(\mathrm{KBr}) v$ $3455 \mathrm{~cm}^{-1}$; HRMS (FAB) (M) calcd for $\mathrm{C}_{26} \mathrm{H}_{42} \mathrm{OS}_{2} 434.2677$, found 434.2686.

7-[ $\alpha$-Hydroxy-(1-naphthylmethyl)]-7-isopropyl-8,11-dithiapentadec-5-yne (3d): 87\% (dr ratio $=6.0 / 1)$; major isomer ${ }^{1} \mathrm{H}$ NMR $\left(\mathrm{CDCl}_{3}, 400 \mathrm{MHz}\right) \delta 0.72-1.12(\mathrm{~m}, 6 \mathrm{H}), 1.17-1.58(\mathrm{~m}, 11 \mathrm{H})$, $1.76-1.90(\mathrm{~m}, 2 \mathrm{H}), 2.08-2.62(\mathrm{~m}, 6 \mathrm{H}), 3.22(\mathrm{~d}, J=8.6 \mathrm{~Hz}, 1$ $\mathrm{H}), 5.86(\mathrm{~d}, J=8.6 \mathrm{~Hz}, 1 \mathrm{H}), 7.40-7.52(\mathrm{~m}, 3 \mathrm{H}), 7.77-7.86(\mathrm{~m}$, $2 \mathrm{H}), 7.93-7.98(\mathrm{~d}, J=7.6 \mathrm{~Hz}, 1 \mathrm{H}), 8.18-8.24(\mathrm{~m}, 1 \mathrm{H})$; characteristic ${ }^{1} \mathrm{H}$ NMR signals for the minor isomer $\delta 2.77 \mathrm{~d}, J=$ $6.0 \mathrm{~Hz}, 1 \mathrm{H}), 8.37(\mathrm{~d}, J=8.0 \mathrm{~Hz}, 1 \mathrm{H})$; IR $(\mathrm{KBr}) v 3426 \mathrm{~cm}^{-1}$; HRMS (FAB) $(\mathrm{M}+\mathrm{H})$ calcd for $\mathrm{C}_{27} \mathrm{H}_{39} \mathrm{OS}_{2}$ 443.2441, found 443.2442 .

7-( $\alpha$-Hydroxy-4-methoxybenzyl)-7-isopropyl-8,11-dithiapentadec-5-yne (3e): $80 \%(\mathrm{dr}$ ratio $=6.4 / 1)$; major isomer ${ }^{1} \mathrm{H} \mathrm{NMR}$ $\left(\mathrm{CDCl}_{3}, 400 \mathrm{MHz}\right) \delta 0.92(\mathrm{t}, J=7.0 \mathrm{~Hz}, 3 \mathrm{H}), 0.94(\mathrm{t}, J=7.0$ $\mathrm{Hz}, 3 \mathrm{H}), 1.11(\mathrm{~d}, J=6.4, \mathrm{~Hz}, 6 \mathrm{H}), 1.34-1.47$ (m, $4 \mathrm{H}), 1.48-1.58$ $(\mathrm{m}, 4 \mathrm{H}), 1.84$ (sept, $J=6.6 \mathrm{~Hz}, 1 \mathrm{H}), 2.29(\mathrm{t}, J=7.0 \mathrm{~Hz}, 2 \mathrm{H})$, $2.47(\mathrm{t}, J=7.4 \mathrm{~Hz}, 2 \mathrm{H}), 2.51-2.72(\mathrm{~m}, 4 \mathrm{H}), 3.04(\mathrm{~d}, J=6.0$ $\mathrm{Hz}, 1 \mathrm{H}), 3.82(\mathrm{~s}, 3 \mathrm{H}), 4.74(\mathrm{~d}, J=6.0 \mathrm{~Hz}, 1 \mathrm{H}), 6.82-6.88(\mathrm{~m}$, $2 \mathrm{H}), 7.42-7.47(\mathrm{~m}, 2 \mathrm{H})$; characteristic ${ }^{1} \mathrm{H}$ NMR signals for the minor isomer $\delta 3.90(\mathrm{~s}, 3 \mathrm{H}), 4.78(\mathrm{~d}, J=4.8 \mathrm{~Hz}, 1 \mathrm{H})$; IR $(\mathrm{KBr})$ $v 3467 \mathrm{~cm}^{-1}$; HRMS $\left(\mathrm{EI}^{+}\right)(\mathrm{M})$ calcd for $\mathrm{C}_{24} \mathrm{H}_{38} \mathrm{O}_{2} \mathrm{~S}_{2} 422.2313$, found 422.2309 .

7-( $\alpha$-Hydroxy-4-bromobenzyl)-7-isopropyl-8,11-dithiapentadec5-yne (3f): $56 \%(\mathrm{dr}$ ratio $=5.4 / 1)$; major isomer ${ }^{1} \mathrm{H} \mathrm{NMR}\left(\mathrm{CDCl}_{3}\right.$, $400 \mathrm{MHz}) \delta 0.92(\mathrm{t}, J=7.4 \mathrm{~Hz}, 3 \mathrm{H}), 0.94(\mathrm{t}, J=7.2 \mathrm{~Hz}, 3 \mathrm{H})$, $1.11(\mathrm{~d}, J=6.6, \mathrm{~Hz}, 3 \mathrm{H}), 1.13(\mathrm{~d}, J=6.6 \mathrm{~Hz}, 3 \mathrm{H}), 1.35-1.46$ $(\mathrm{m}, 4 \mathrm{H}), 1.47-1.57(\mathrm{~m}, 4 \mathrm{H}), 1.84$ (sept, $J=6.6 \mathrm{~Hz}, 1 \mathrm{H}), 2.27$ $(\mathrm{t}, J=7.0 \mathrm{~Hz}, 2 \mathrm{H}), 2.46(\mathrm{t}, J=7.2 \mathrm{~Hz}, 2 \mathrm{H}), 2.49-2.68(\mathrm{~m}, 4$ $\mathrm{H}), 3.11(\mathrm{~d}, J=6.8 \mathrm{~Hz}, 1 \mathrm{H}), 4.76(\mathrm{~d}, J=6.8 \mathrm{~Hz}, 1 \mathrm{H}), 7.38-7.45$ $(\mathrm{m}, 4 \mathrm{H})$; characteristic ${ }^{1} \mathrm{H}$ NMR signals for the minor isomer $\delta$ $2.32(\mathrm{t}, J=7.0 \mathrm{~Hz}, 2 \mathrm{H}), 3.04(\mathrm{~d}, J=4.8 \mathrm{~Hz}, 1 \mathrm{H}), 4.78(\mathrm{~d}, J=$ $4.8 \mathrm{~Hz}, 1 \mathrm{H})$; IR (KBr) $v 3405 \mathrm{~cm}^{-1}$; HRMS (FAB) $(\mathrm{M}+\mathrm{H})^{+}$ calcd for $\mathrm{C}_{23} \mathrm{H}_{36} \mathrm{OBrS} \mathrm{S}_{2}$ 471.1391, found 471.1389.

3-( $\alpha$-Hydroxybenzyl)-3-isopropyl-1-trimethylsilyl-4,7-dithiaundec-1-yne $(3 \mathrm{~g})$ : $83 \%(\mathrm{dr}$ ratio $=4.6 / 1)$; major isomer ${ }^{1} \mathrm{H}$ NMR $\left(\mathrm{CDCl}_{3}, 400 \mathrm{MHz}\right) \delta 0.19(\mathrm{~s}, 9 \mathrm{H}), 0.90(\mathrm{t}, J=6.4 \mathrm{~Hz}, 3 \mathrm{H})$, $1.08-1.20(\mathrm{~m}, 6 \mathrm{H}), 1.36-1.42(\mathrm{~m}, 2 \mathrm{H}), 1.44-1.58(\mathrm{~m}, 2 \mathrm{H})$, 1.87 (sept, $J=6.6 \mathrm{~Hz}, 1 \mathrm{H}), 2.40-2.64(\mathrm{~m}, 6 \mathrm{H}), 2.41-2.68(\mathrm{~m}$, $6 \mathrm{H}), 3.08(\mathrm{~d}, J=6.8 \mathrm{~Hz}, 1 \mathrm{H}), 4.82(\mathrm{~d}, J=6.8 \mathrm{~Hz}, 1 \mathrm{H})$, 7.26-7.34 (m, $3 \mathrm{H}), 7.50-7.60(\mathrm{~m}, 2 \mathrm{H})$; characteristic ${ }^{1} \mathrm{H}$ NMR signals for the minor isomer $\delta 0.23(\mathrm{~s}, 9 \mathrm{H}), 2.96(\mathrm{~d}, J=4.8 \mathrm{~Hz}$, $1 \mathrm{H})^{7}$

3-Butyl-3-( $\alpha$-hydroxybenzyl)-1-trimethylsilyl-4,7-dithiaundec-1yne (3h): $78 \%(\mathrm{dr}$ ratio $=5.3 / 1)$; major isomer ${ }^{1} \mathrm{H} \mathrm{NMR}\left(\mathrm{CDCl}_{3}\right.$, $400 \mathrm{MHz}) \delta 0.20(\mathrm{~s}, 9 \mathrm{H}), 0.84-0.97(\mathrm{~m}, 6 \mathrm{H}), 1.22-1.70(\mathrm{~m}, 8$ $\mathrm{H}), 2.51(\mathrm{t}, J=7.2 \mathrm{~Hz}, 2 \mathrm{H}), 2.60-2.93(\mathrm{~m}, 4 \mathrm{H}), 3.18(\mathrm{~d}, J=4.8$ $\mathrm{Hz}, 1 \mathrm{H}), 4.60$ (d, $J=4.8 \mathrm{~Hz}, 1 \mathrm{H}), 7.28-7.34$ (m, $3 \mathrm{H}), 7.46-7.52$ (m, $2 \mathrm{H})$; characteristic ${ }^{1} \mathrm{H}$ NMR signals for the minor isomer $\delta$ 
$0.21(\mathrm{~s}, 9 \mathrm{H}), 2.56(\mathrm{t}, J=7.4 \mathrm{~Hz}, 2 \mathrm{H}), 3.20(\mathrm{~d}, J=2.2 \mathrm{~Hz}, 1 \mathrm{H})$, $4.71(\mathrm{~d}, J=2.2 \mathrm{~Hz}, 1 \mathrm{H})$; IR (KBr) $v 3458 \mathrm{~cm}^{-1}$; HRMS (FAB) $(\mathrm{M}+\mathrm{H})$ calcd for $\mathrm{C}_{23} \mathrm{H}_{39} \mathrm{OSiS}_{2}$ 423.2212, found 423.2209.

3-Butyl-3-(1-hydroxypentyl)-1-trimethylsilyl-4,7-dithiaundec-1yne (3i): $35 \%(\mathrm{dr}$ ratio $=1.3 / 1)$; major isomer ${ }^{1} \mathrm{H} \mathrm{NMR}\left(\mathrm{CDCl}_{3}\right.$, $400 \mathrm{MHz}) \delta 0.19(\mathrm{~s}, 9 \mathrm{H}), 0.90-0.96(\mathrm{~m}, 9 \mathrm{H}), 1.25-1.87(\mathrm{~m}, 14$ $\mathrm{H}), 2.23(\mathrm{~d}, J=6.2 \mathrm{~Hz}, 1 \mathrm{H}), 2.57(\mathrm{t}, J=7.4 \mathrm{~Hz}, 2 \mathrm{H}), 2.68-3.00$ $(\mathrm{m}, 4 \mathrm{H}), 3.48-3.56(\mathrm{~m}, 2 \mathrm{H})$; characteristic ${ }^{1} \mathrm{H}$ NMR signals for the minor isomer $\delta 0.20(\mathrm{~s}, 9 \mathrm{H})$; IR $(\mathrm{KBr}) v 3484 \mathrm{~cm}^{-1}$; HRMS (FAB) $(\mathrm{M}+\mathrm{H})$ calcd for $\mathrm{C}_{21} \mathrm{H}_{43} \mathrm{OS}_{2} \mathrm{Si} 403.2525$, found 403.2517 .

3-Butyl-3-( $\alpha$-hydroxy-4-trifluoromethylbenzyl)-1-trimethylsilyl4,7-dithiaundec-1-yne $(\mathbf{3 j})$ : $80 \%(\mathrm{dr}$ ratio $=3.6 / 1)$; major isomer ${ }^{1} \mathrm{H} \mathrm{NMR}\left(\mathrm{CDCl}_{3}, 400 \mathrm{MHz}\right) \delta 0.20(\mathrm{~s}, 9 \mathrm{H}), 0.83-0.96(\mathrm{~m}, 6 \mathrm{H})$, $1.19-1.78(\mathrm{~m}, 8 \mathrm{H}), 2.51(\mathrm{t}, J=7.4 \mathrm{~Hz}, 2 \mathrm{H}), 2.63-3.00(\mathrm{~m}, 4$ $\mathrm{H}), 3.33(\mathrm{~d}, J=4.6 \mathrm{~Hz}, 1 \mathrm{H}), 4.63(\mathrm{~d}, J=4.6 \mathrm{~Hz}, 1 \mathrm{H}), 7.57(\mathrm{~d}$, $J=8.4 \mathrm{~Hz}, 2 \mathrm{H}), 7.62(\mathrm{~d}, J=8.4 \mathrm{~Hz}, 2 \mathrm{H})$; characteristic ${ }^{1} \mathrm{H}$ NMR signals for the minor isomer $\delta 0.21(\mathrm{~s}, 9 \mathrm{H}), 3.45-3.52(\mathrm{~m}$, $1 \mathrm{H}), 4.76$ (br s, $1 \mathrm{H}$ ); IR (KBr) $v 3449 \mathrm{~cm}^{-1}$; HRMS (FAB) (M $+\mathrm{H})$ calcd for $\mathrm{C}_{24} \mathrm{H}_{38} \mathrm{OF}_{3} \mathrm{SiS}_{2}: 491.2085$, found 491.2079.

3-Butyl-3-( $\alpha$-hydroxy-4-cyanobenzyl)-1-trimethylsilyl-4,7-dithiaundec-1-yne (3k): $83 \%(\mathrm{dr}$ ratio $=1.6 / 1)$. The two isomers were separated by preparative HPLC (LiChrospher Si 60, eluent: $\mathrm{CHCl}_{3} /$ EtOAc $=100 / 1)$ : major isomer $(3 \mathbf{k b}){ }^{1} \mathrm{H} \mathrm{NMR}\left(\mathrm{CDCl}_{3}, 400 \mathrm{MHz}\right)$ $\delta 0.18(\mathrm{~s}, 9 \mathrm{H}), 0.90(\mathrm{t}, J=8.0 \mathrm{~Hz}, 3 \mathrm{H}), 0.91(\mathrm{t}, J=7.4 \mathrm{~Hz}, 3$ $\mathrm{H}), 1.16-1.66(\mathrm{~m}, 8 \mathrm{H}), 2.52(\mathrm{t}, J=7.6 \mathrm{~Hz}, 2 \mathrm{H}), 2.62-3.01(\mathrm{~m}$, $4 \mathrm{H}), 3.37(\mathrm{~d}, J=4.4 \mathrm{~Hz}, 1 \mathrm{H}), 4.61(\mathrm{~d}, J=4.4 \mathrm{~Hz}, 1 \mathrm{H})$, 7.59-7.64 (m, $4 \mathrm{H})$; minor isomer (3ka) ${ }^{1} \mathrm{H}$ NMR $\left(\mathrm{CDCl}_{3}, 400\right.$ $\mathrm{MHz}) \delta 0.20(\mathrm{~s}, 9 \mathrm{H}), 0.84(\mathrm{t}, J=7.2 \mathrm{~Hz}, 3 \mathrm{H}), 0.93(\mathrm{t}, J=7.4$ $\mathrm{Hz}, 3 \mathrm{H}), 1.18-1.66(\mathrm{~m}, 8 \mathrm{H}), 2.57(\mathrm{t}, J=7.2 \mathrm{~Hz}, 2 \mathrm{H}), 2.62-3.01$ $(\mathrm{m}, 4 \mathrm{H}), 3.52(\mathrm{~d}, J=1.6 \mathrm{~Hz}, 1 \mathrm{H}), 4.73(\mathrm{~d}, J=1.6 \mathrm{~Hz}, 1 \mathrm{H})$, 7.59-7.64 (m, $4 \mathrm{H})$; IR (KBr) $v 3471 \mathrm{~cm}^{-1}$; HRMS (FAB) $(\mathrm{M}+$ $\mathrm{H})$ calcd for $\mathrm{C}_{24} \mathrm{H}_{38} \mathrm{ONSiS}_{2} 448.2164$, found 448.2175.

3-Butyl-3-( $\alpha$-hydroxy- $o$-methylbenzyl)-1-trimethylsilyl-4,7-dithiaundec-1-yne (3l): $67 \%(\mathrm{dr}$ ratio $=1.7 / 1)$; major isomer ${ }^{1} \mathrm{H}$ NMR $\left(\mathrm{CDCl}_{3}, 400 \mathrm{MHz}\right) \delta 0.18(\mathrm{~s}, 9 \mathrm{H}), 0.85-0.95(\mathrm{~m}, 6 \mathrm{H}), 1.24-1.66$ $(\mathrm{m}, 8 \mathrm{H}), 2.40(\mathrm{~s}, 3 \mathrm{H}), 2.46-2.88(\mathrm{~m}, 8 \mathrm{H}), 3.23(\mathrm{~d}, J=5.2 \mathrm{~Hz}$, $1 \mathrm{H}), 4.89(\mathrm{~d}, J=5.2 \mathrm{~Hz}, 1 \mathrm{H}), 7.09-7.16(\mathrm{~m}, 1 \mathrm{H}), 7.17-7.22$ (m, $2 \mathrm{H}), 7.77-7.82(\mathrm{~m}, 1 \mathrm{H})$; characteristic ${ }^{1} \mathrm{H}$ NMR signals for the minor isomer $\delta 0.18(\mathrm{~s}, 9 \mathrm{H}), 2.94(\mathrm{~d}, J=4.0 \mathrm{~Hz}, 1 \mathrm{H}), 5.04$ $(\mathrm{d}, J=4.0 \mathrm{~Hz}, 1 \mathrm{H}), 7.60-7.65(\mathrm{~m}, 2 \mathrm{H})$; IR (KBr) P $3455 \mathrm{~cm}^{-1}$; HRMS (FAB) $(\mathrm{M}+\mathrm{H})^{+}$calcd for $\mathrm{C}_{24} \mathrm{H}_{41} \mathrm{OSiS}_{2} 437.2368$, found 437.2370.

7-Butyl-7-( $\alpha$-hydroxy-4-(tert-butoxycarbonyl)benzyl)-8,11-dithiapentadec-5-yne (3m). Under $\mathrm{N}_{2}$ atmosphere, to a DCM solution $(45 \mathrm{~mL})$ of 4-carboxybenzaldehyde $(4.5 \mathrm{~g}, 30 \mathrm{mmol})$, imidazole (4.08 g, $60 \mathrm{mmol})$, 4-dimethylaminopyridine $(0.37 \mathrm{~g}, 3 \mathrm{mmol})$, and tert-butanol $(8.6 \mathrm{~mL}, 90 \mathrm{mmol})$ cooled at $0{ }^{\circ} \mathrm{C}$ was added a DCM solution $(15 \mathrm{~mL})$ of DCC (6.81 g, $33 \mathrm{mmol}, 1.1$ equiv) dropwise. The mixture was stirred for $3 \mathrm{~h}$ at $\mathrm{rt}$ and filtered. The filtrate was washed with $0.5 \mathrm{~N} \mathrm{HCl}_{(\mathrm{aq})}$ and $\mathrm{NaHCO}_{3 \text { (sat.) }}$. The organic layer was dried $\left(\mathrm{MgSO}_{4}\right)$ and filtered and the filtrate was evaporated in vacuo to give the residue, which was chromatographed on silica gel (hexane $/ \mathrm{CHCl}_{3}=1 / 1$ ) to give tert-butyl 4-formylbenzoate as a white solid (2.91 g, 49\%). Mp 45-46 ${ }^{\circ} \mathrm{C} ;{ }^{1} \mathrm{H}$ NMR $\left(\mathrm{CDCl}_{3}, 400\right.$ $\mathrm{MHz}) \delta 1.60(\mathrm{~s}, 9 \mathrm{H}), 7.90(\mathrm{~d}, J=8.0 \mathrm{~Hz}, 2 \mathrm{H}), 8.11(\mathrm{~d}, J=8.0$ $\mathrm{Hz}, 2 \mathrm{H}), 10.06(\mathrm{~s}, 1 \mathrm{H}) .^{20}$

3m: $37 \%(\mathrm{dr}$ ratio $=1.7 / 1)$; major isomer ${ }^{1} \mathrm{H} \mathrm{NMR}\left(\mathrm{CDCl}_{3}\right.$, $400 \mathrm{MHz}) \delta 0.82-1.00(\mathrm{~m}, 9 \mathrm{H}), 1.18-1.68(\mathrm{~m}, 17 \mathrm{H}), 2.27(\mathrm{t}, J$ $=7.0 \mathrm{~Hz}, 2 \mathrm{H}), 2.50(\mathrm{t}, J=7.4 \mathrm{~Hz}, 2 \mathrm{H}), 2.63-2.88(\mathrm{~m}, 4 \mathrm{H})$, $3.32(\mathrm{~d}, J=4.4 \mathrm{~Hz}, 1 \mathrm{H}), 4.60,(\mathrm{~d}, J=4.4 \mathrm{~Hz}, 1 \mathrm{H}), 7.51-7.55$ $(\mathrm{m}, 4 \mathrm{H}), 7.7 .91-7.95(\mathrm{~m}, 2 \mathrm{H})$; characteristic ${ }^{1} \mathrm{H}$ NMR signals for the minor isomer $\delta 3.46(\mathrm{~d}, J=1.8 \mathrm{~Hz}, 1 \mathrm{H}), 4.73(\mathrm{~d}, J=1.8$ $\mathrm{Hz}, 1 \mathrm{H})$; IR $(\mathrm{KBr}) v 3471 \mathrm{~cm}^{-1}$; HRMS (FAB) $(\mathrm{M}+\mathrm{H})$ calcd for $\mathrm{C}_{29} \mathrm{H}_{47} \mathrm{O}_{3} \mathrm{~S}_{2}$ 507.2967, found 507.2959.

(20) Kangn, S. U.; Choi, W. J.; Oishi, S.; Lee, K.; Karki, R. G.; Worthy, K. M.; Bindu, L. K.; Nicklaus, M. C.; Fisher, R. J.; Burke, T. R., Jr J. Med. Chem. 2007, 50, 1978.
7-Butyl-7-(2-hydroxy-2-propyl)-8,11-dithiapentadec-5-yne (3n): $53 \% ;{ }^{1} \mathrm{H} \mathrm{NMR}\left(\mathrm{CDCl}_{3}, 400 \mathrm{MHz}\right) \delta 0.82-0.96(\mathrm{~m}, 9 \mathrm{H}), 1.2-1.68$ $(\mathrm{m}, 19 \mathrm{H}), 1.79(\mathrm{dt}, J=4.1,12 \mathrm{~Hz}, 1 \mathrm{H}), 2.26(\mathrm{t}, J=7.0 \mathrm{~Hz}, 2$ $\mathrm{H}), 2.41(\mathrm{~s}, 1 \mathrm{H}), 2.52(\mathrm{t}, J=7.4 \mathrm{~Hz}, 2 \mathrm{H}), 2.63-2.78(\mathrm{~m}, 2 \mathrm{H})$, 2.83-2.96 (m, $2 \mathrm{H}) ;{ }^{13} \mathrm{C}$ NMR $\left(100 \mathrm{MHz}, \mathrm{CDCl}_{3}\right) \delta$ 13.6, 13.7, $14.2,18.6,22.0,22.1,22.9,25.5,28.8,31.0,31.8,31.9,32.9,33.2$, 36.7, 60.6, 76.1, 78.1, 89.4; IR (KBr) $v 3487 \mathrm{~cm}^{-1}$; HRMS (EI $\left.{ }^{+}\right)$ (M) calcd for $\mathrm{C}_{20} \mathrm{H}_{38} \mathrm{OS}_{2} 358.2364$, found 358.2353.

7-Butyl-7-(1-hydroxy-1,1-diphenylmethyl)-8,11-dithiapentadec5-yne (3o): 78\%; ${ }^{1} \mathrm{H} \mathrm{NMR}\left(\mathrm{CDCl}_{3}, 400 \mathrm{MHz}\right) \delta 0.85$ (t, $J=7.2$ $\mathrm{Hz}, 3 \mathrm{H}), 0.91(\mathrm{t}, J=7.2 \mathrm{~Hz}, 3 \mathrm{H}), 0.93(\mathrm{t}, J=7.2 \mathrm{~Hz}, 3 \mathrm{H})$, $1.23-1.62(\mathrm{~m}, 12 \mathrm{H}), 1.95-2.19(\mathrm{~m}, 4 \mathrm{H}), 2.47-2.58(\mathrm{~m}, 4 \mathrm{H})$, 2.63-2.79 (m, $2 \mathrm{H}), 2.98$ (s, $1 \mathrm{H}), 7.21-7.44(\mathrm{~m}, 10 \mathrm{H}) ;{ }^{13} \mathrm{C} \mathrm{NMR}$ $\left(100 \mathrm{MHz}, \mathrm{CDCl}_{3}\right) \delta 13.8,14.0,14.1,22.1,22.4,22.7,29.9,30.6$, 30.7, 31.7, 31.8, 31.9, 32.0, 33.8, 81.3, 108.1, 118.0, 127.0, 127.2, 127.7, 145.0, 145.1; IR (KBr) P3488 cm $\mathrm{cm}^{-1}$; HRMS (FAB) $(\mathrm{M}+$ H) calcd for $\mathrm{C}_{30} \mathrm{H}_{42} \mathrm{OS}_{2}$ 483.2755, found 483.2756.

4-Isopropyl-4-( $\alpha$-hydroxybenzyl)-5,8-dithia-2-dodecyn-1-yl tertButyldimethylsilyl Ether (3p). Under $\mathrm{N}_{2}$ atmosphere, to a THF solution $(50 \mathrm{~mL})$ of $1 \mathrm{e}(1.01 \mathrm{~g}, 5.0 \mathrm{mmol}, 1.0$ equiv) cooled at -78 ${ }^{\circ} \mathrm{C}$ was added dropwise BuLi (4.4 mL, 2.2 equiv, $2.5 \mathrm{M}$ hexane solution). After the solution was stirred for $1 \mathrm{~h}$, benzaldehyde $(0.51$ $\mathrm{mL}, 5.0 \mathrm{mmol}, 1.0$ equiv) in THF $(10 \mathrm{~mL})$ was added dropwise at $-78{ }^{\circ} \mathrm{C}$. The mixture was gradually warmed to rt and stirred for $8 \mathrm{~h}$, quenched with sat. $\mathrm{NH}_{4} \mathrm{Cl}$, washed with brine, and extracted with ether. The organic layer was dried $\left(\mathrm{MgSO}_{4}\right)$ and filtered and the filtrate was evaporated in vacuo to give the residue, which was chromatographed on silica gel (hexane/EtOAc $=9 / 1$ ) to give the crude diol $(1.47 \mathrm{~g}, 77 \%)$.

Under $\mathrm{N}_{2}$ atmosphere, to a DCM solution $(40 \mathrm{~mL})$ of the crude diol (1.47 g, $3.85 \mathrm{mmol})$ and imidazole $(0.26 \mathrm{~g}, 3.85 \mathrm{mmol}, 1.0$ equiv) cooled at $-78{ }^{\circ} \mathrm{C}$ was added TBSCl $(0.58 \mathrm{~g}, 3.85 \mathrm{mmol}$, 1.0 equiv) in DCM (10 mL) dropwise. The mixture was gradually warmed to rt and stirred for $8 \mathrm{~h}$ and then washed with brine. The organic layer was dried $\left(\mathrm{MgSO}_{4}\right)$ and filtered and the filtrate was evaporated in vacuo to give the residue, which was chromatographed on silica gel (hexane/EtOAc $=30 / 1$ ) to give $\mathbf{3 p}$ as a pale yellow liquid $(1.51 \mathrm{~g}, 63 \%$ in two steps, dr ratio $=3.5 / 1)$. Major isomer ${ }^{1} \mathrm{H} \mathrm{NMR}\left(\mathrm{CDCl}_{3}, 400 \mathrm{MHz}\right) \delta 0.12(\mathrm{~s}, 6 \mathrm{H}), 0.88-0.98$ $\left(\mathrm{m}, 12 \mathrm{H}\right.$ embodied a singlet at 0.93 for ${ }^{t} \mathrm{Bu}$ group), $1.14(\mathrm{~d}, J=$ $6.7 \mathrm{~Hz}, 6 \mathrm{H}), 1.33-1.42(\mathrm{~m}, 2 \mathrm{H}), 1.47-1.56$ (m, $2 \mathrm{H}), 1.89$ (sept, $J=6.7 \mathrm{~Hz}, 1 \mathrm{H}), 2.41-2.70(\mathrm{~m}, 6 \mathrm{H}), 3.10(\mathrm{~s}, 1 \mathrm{H}), 4.40(\mathrm{~s}, 2 \mathrm{H})$, $4.84(\mathrm{~s}, 1 \mathrm{H}), 7.26-7.36(\mathrm{~m}, 3 \mathrm{H}), 7.50-7.60(\mathrm{~m}, 2 \mathrm{H})$; characteristic ${ }^{1} \mathrm{H}$ NMR signals for the minor isomer $\delta 0.14(\mathrm{~s}, 6$ H), 2.19 (sept, $J=6.8 \mathrm{~Hz}, 1 \mathrm{H}$ ), 4.43 (s, 2 H); IR (KBr) P 3459 $\mathrm{cm}^{-1}$; HRMS (FAB) $(\mathrm{M}+\mathrm{H})$ calcd for $\mathrm{C}_{26} \mathrm{H}_{45} \mathrm{O}_{2} \mathrm{SiS}_{2} 481.2630$, found 481.2632.

General Procedure for the Preparation of Enyne 4. Under $\mathrm{N}_{2}$ atmosphere, to a THF solution $(5 \mathrm{~mL})$ of $\mathbf{3}(0.5 \mathrm{mmol}, 1.0$ equiv) and $\mathrm{Ph}_{3} \mathrm{P}(0.26 \mathrm{~g}, 1.0 \mathrm{mmol}, 2.0$ equiv) was added DIAD (0.20 $\mathrm{mL} 1.0 \mathrm{mmol}, 2.0$ equiv) at rt. After being stirred for $18 \mathrm{~h}$, the mixture was evaporated in vacuo to give the residue, which was chromatographed on silica gel (hexane/ $\mathrm{CHCl}_{3}=1 / 0$ to $10 / 1$ ) to give 4.

2-Isopropyl-1-phenyloct-1E-en-3-yne (4a): $81 \%(E / Z=3.0 / 1)$; $E$ isomer ${ }^{1} \mathrm{H} \mathrm{NMR}\left(\mathrm{CDCl}_{3}, 400 \mathrm{MHz}\right) \delta 0.97(\mathrm{t}, J=7.4 \mathrm{~Hz}, 3 \mathrm{H})$, $1.13(\mathrm{~d}, J=6.4 \mathrm{~Hz}, 6 \mathrm{H}), 1.44-1.66(\mathrm{~m}, 4 \mathrm{H}), 2.40(\mathrm{t}, J=6.8$ $\mathrm{Hz}, 2 \mathrm{H}$ ), 3.04 (sept, $J=6.6 \mathrm{~Hz}, 1 \mathrm{H}$ ), 6.70 (s, $1 \mathrm{H}), 7.20-7.36$ $(\mathrm{m}, 5 \mathrm{H})$; characteristic ${ }^{1} \mathrm{H}$ NMR signals for $Z$ isomer $\delta 2.48(\mathrm{t}, J$ $=7.0 \mathrm{~Hz}, 2 \mathrm{H}), 2.55$ (sept, $J=6.8 \mathrm{~Hz}, 1 \mathrm{H}), 6.49(\mathrm{~s}, 1 \mathrm{H}), 7.82$ $(\mathrm{d}, J=7.6 \mathrm{~Hz}, 2 \mathrm{H})$; HRMS (FAB) (M) calcd for $\mathrm{C}_{17} \mathrm{H}_{22} 226.1722$, found 226.1721 .

6-Isopropyldodec-5E-en-7-yne (4b): $43 \%(E / Z=10 / 1) ; E$ isomer ${ }^{1} \mathrm{H} \mathrm{NMR}\left(\mathrm{CDCl}_{3}, 400 \mathrm{MHz}\right) \delta 0.89(\mathrm{t}, J=7.2 \mathrm{~Hz}, 3 \mathrm{H}), 0.93(\mathrm{t}$, $J=7.2 \mathrm{~Hz}, 3 \mathrm{H}), 1.04(\mathrm{~d}, J=7.0 \mathrm{~Hz}, 6 \mathrm{H}), 1.28-1.38(\mathrm{~m}, 4 \mathrm{H})$, $1.39-1.57(\mathrm{~m}, 4 \mathrm{H}), 2.09(\mathrm{td}, J=7.4,6.8 \mathrm{~Hz}), 2.32(\mathrm{t}, J=7.0$ $\mathrm{Hz}, 2 \mathrm{H}), 2.73$ (sept, $J=7.0 \mathrm{~Hz}, 1 \mathrm{H}), 5.66(\mathrm{t}, J=7.4 \mathrm{~Hz}, 1 \mathrm{H})$; ${ }^{13} \mathrm{C}$ NMR $\left(100 \mathrm{MHz}, \mathrm{CDCl}_{3}\right) \delta 14.1,14.4,19.4,21.9,22.4,22.8$, 27.9, 28.4, 31.5, 32.0, 79.9, 88.3, 129.6, 134.2; characteristic ${ }^{1} \mathrm{H}$ 
NMR signals for $Z$ isomer $\delta 5.58(\mathrm{t}, J=7.0 \mathrm{~Hz}, 1 \mathrm{H})$; HRMS (FAB) (M) calcd for $\mathrm{C}_{15} \mathrm{H}_{26}$ 206.2035, found 206.2040.

2-Isopropyl-1-mesityloct-1Z-en-3-yne (4c): $65 \%(E / Z=1 / 20)$; characteristic ${ }^{1} \mathrm{H}$ NMR signals for $E$ isomer $\delta 0.85(\mathrm{t}, J=7.4 \mathrm{~Hz}$, $3 \mathrm{H}), 6.61(\mathrm{~s}, 1 \mathrm{H}) ; Z$ isomer ${ }^{1} \mathrm{H}$ NMR $\left(\mathrm{CDCl}_{3}, 400 \mathrm{MHz}\right) \delta 1.01$ $(\mathrm{t}, J=7.4 \mathrm{~Hz}, 3 \mathrm{H}), 1.07(\mathrm{~d}, J=6.8 \mathrm{~Hz}, 6 \mathrm{H}), 1.41-1.53(\mathrm{~m}, 2$ $\mathrm{H}), 1.61-1.70(\mathrm{~m}, 2 \mathrm{H}), 2.28(\mathrm{~s}, 6 \mathrm{H}), 2.31(\mathrm{~s}, 3 \mathrm{H}), 2.33(\mathrm{t}, J=$ $7.4 \mathrm{~Hz}, 2 \mathrm{H}$ ), 2.55 (sept, $J=6.8 \mathrm{~Hz}, 1 \mathrm{H}), 6.46$ (s, $1 \mathrm{H}), 6.87$ (s, $2 \mathrm{H}) ;{ }^{13} \mathrm{C} \mathrm{NMR}\left(100 \mathrm{MHz}, \mathrm{CDCl}_{3}\right) \delta 14.4,20.7,21.4,21.5,22.5$, 23.3, 31.1, 37.8, 79.0, 99.6, 126.5, 127.3, 132.2, 133.6, 135.3, 135.7; HRMS (FAB) (M) calcd for $\mathrm{C}_{10} \mathrm{H}_{28} 268.2191$, found 268.2188.

2-Isopropyl-1(1-naphthyl)-oct-1-Z-en-3-yne (4d): $72 \%(E / Z=$ 1/3.9); characteristic ${ }^{1} \mathrm{H}$ NMR signals for $E$ isomer $\delta 0.89(\mathrm{t}, J=$ $7.2 \mathrm{~Hz}, 3 \mathrm{H}), 1.28$ (d, $J=6.8 \mathrm{~Hz}, 6 \mathrm{H}), 2.32$ (t, $J=6.8 \mathrm{~Hz}, 2 \mathrm{H})$, 2.70 (sept, $J=6.8 \mathrm{~Hz}, 1 \mathrm{H}), 7.14(\mathrm{~s}, 1 \mathrm{H}) ; Z$ isomer $\delta 1.00(\mathrm{t}, J$ $=7.2 \mathrm{~Hz}, 3 \mathrm{H}), 1.09(\mathrm{~d}, J=6.6 \mathrm{~Hz}, 6 \mathrm{H}), 1.50-1.69(\mathrm{~m}, 4 \mathrm{H})$, $2.46(\mathrm{t}, J=7.0 \mathrm{~Hz}, 2 \mathrm{H}), 2.79$ (sept, $J=6.6 \mathrm{~Hz}, 1 \mathrm{H}), 7.09$ (s, 1 H), $7.30(\mathrm{~d}, J=7.2 \mathrm{~Hz}, 1 \mathrm{H}), 7.42-7.54(\mathrm{~m}, 3 \mathrm{H}), 7.78(\mathrm{~d}, J=$ $8.0 \mathrm{~Hz}, 1 \mathrm{H}), 7.81-7.89(\mathrm{~m}, 1 \mathrm{H}), 7.97-8.07$ (m, 1 H); HRMS (FAB) (M) calcd for $\mathrm{C}_{17} \mathrm{H}_{22} 276.1878$, found 276.1875 .

2-Isopropyl-1-(4-methoxyphenyl)oct-1E-en-3-yne (4e): $91 \%(E / Z$ $=5.0 / 1) ; E$ isomer ${ }^{1} \mathrm{H} \mathrm{NMR}\left(\mathrm{CDCl}_{3}, 400 \mathrm{MHz}\right) \delta 0.96(\mathrm{t}, J=7.2$ $\mathrm{Hz}, 3 \mathrm{H}), 1.13(\mathrm{~d}, J=6.7 \mathrm{~Hz}, 6 \mathrm{H}), 1.40-1.66(\mathrm{~m}, 4 \mathrm{H}), 2.39$ (t, $J=6.8 \mathrm{~Hz}, 2 \mathrm{H}), 3.04$ (sept, $J=6.7 \mathrm{~Hz}, 1 \mathrm{H}), 3.82$ (s, $3 \mathrm{H}), 6.64$ (s, $1 \mathrm{H}), 6.82-6.89(\mathrm{~m}, 2 \mathrm{H}), 7.14-7.19(\mathrm{~m}, 2 \mathrm{H})$; characteristic ${ }^{1} \mathrm{H}$ NMR signals for $Z$ isomer $\delta 3.80(\mathrm{~s}, 3 \mathrm{H}), 6.43(\mathrm{~s}, 1 \mathrm{H})$, 7.78-7.81 (m, $2 \mathrm{H})$; HRMS (FAB) (M) calcd for $\mathrm{C}_{18} \mathrm{H}_{24} \mathrm{O}$ 256.1827 , found 256.1831 .

1-(4-Bromophenyl)-2-isopropyl-oct-1E-en-3-yne (4f): 68\% (E/Z $=1.7 / 1) ; E$ isomer ${ }^{1} \mathrm{H} \mathrm{NMR}\left(\mathrm{CDCl}_{3}, 400 \mathrm{MHz}\right) \delta 0.94(\mathrm{t}, J=7.4$ $\mathrm{Hz}, 3 \mathrm{H}), 1.10(\mathrm{~d}, J=6.5 \mathrm{~Hz}, 6 \mathrm{H}), 1.44-1.61(\mathrm{~m}, 4 \mathrm{H}), 2.38$ (t, $J=7.0 \mathrm{~Hz}, 2 \mathrm{H}), 2.95$ (sept, $J=6.5 \mathrm{~Hz}, 1 \mathrm{H}), 6.58(\mathrm{~s}, 1 \mathrm{H})$, 7.04-7.08 (m, $2 \mathrm{H}), 7.40-7.44(\mathrm{~m}, 2 \mathrm{H}) ;{ }^{13} \mathrm{C} \mathrm{NMR}(100 \mathrm{MHz}$, $\left.\mathrm{CDCl}_{3}\right) \delta 13.8,19.3,21.8,22.1,28.8,31.1,79.9,92.0,120.6,130.1$, 131.2, 131.3, 133.5, 135.8; characteristic ${ }^{1} \mathrm{H}$ NMR signals for $Z$ isomer $\delta 2.47(\mathrm{t}, J=7.0 \mathrm{~Hz}, 2 \mathrm{H}), 2.53(\mathrm{sept}, J=6.8 \mathrm{~Hz}, 1 \mathrm{H})$, $6.42(\mathrm{~s}, 1 \mathrm{H}), 7.67-7.71(\mathrm{~m}, 2 \mathrm{H})$; HRMS (FAB) (M) calcd for $\mathrm{C}_{17} \mathrm{H}_{21}{ }^{79} \mathrm{Br} 304.0827$, found 304.0824 .

2-Isopropyl-1-phenyl-4-trimethylsilylbut-1E-en-3-yne (4g): 78\% $(E / Z=3.2 / 1) ; E$ isomer ${ }^{1} \mathrm{H} \mathrm{NMR}\left(\mathrm{CDCl}_{3}, 400 \mathrm{MHz}\right) \delta 0.25$ (s, 9 H), 1.15 (d, $J=6.8 \mathrm{~Hz}, 6 \mathrm{H}), 3.04$ (sept, $J=6.8 \mathrm{~Hz}, 1 \mathrm{H}), 6.83$ (s, $1 \mathrm{H}), 7.20-7.37(\mathrm{~m}, 5 \mathrm{H})$; characteristic ${ }^{1} \mathrm{H}$ NMR signals for $Z$ isomer $\delta 0.26(\mathrm{~s}, 9 \mathrm{H}), 1.19(\mathrm{~d}, J=6.4 \mathrm{~Hz}, 6 \mathrm{H}), 2.56$ (sept, $J=$ $6.1 \mathrm{~Hz}, 1 \mathrm{H}), 6.57(\mathrm{~s}, 1 \mathrm{H}), 7.86(\mathrm{~d}, J=7.6 \mathrm{~Hz}, 2 \mathrm{H}){ }^{7}$

(3-Butyl-4-phenylbut-3E-en-1-yn-1-yl)trimethylsilane (4h): $72 \%$ $(E / Z=13.5 / 1) ; E$ isomer ${ }^{1} \mathrm{H}$ NMR $\left(\mathrm{CDCl}_{3}, 400 \mathrm{MHz}\right) \delta 0.26(\mathrm{~s}$, $9 \mathrm{H}), 0.93(\mathrm{t}, J=7.4 \mathrm{~Hz}, 3 \mathrm{H}), 1.33-1.44(\mathrm{~m}, 2 \mathrm{H}), 1.58-1.79$ $(\mathrm{m}, 2 \mathrm{H}), 2.39(\mathrm{t}, J=7.6 \mathrm{~Hz}, 2 \mathrm{H}), 6.92(\mathrm{~s}, 1 \mathrm{H}), 7.22-7.30(\mathrm{~m}$, $3 \mathrm{H}), 7.31-7.38(\mathrm{~m}, 2 \mathrm{H}) ;{ }^{13} \mathrm{C} \mathrm{NMR}\left(100 \mathrm{MHz}, \mathrm{CDCl}_{3}\right) \delta 0.24$, 14.1, 22.5, 30.7, 31.0, 93.7, 107.7, 125.4, 127.0, 128.1, 128.7, 136.5; characteristic ${ }^{1} \mathrm{H}$ NMR signals for $Z$ isomer $\delta 0.29(\mathrm{~s}, 9 \mathrm{H}), 2.32$ $(\mathrm{t}, J=7.6 \mathrm{~Hz}, 2 \mathrm{H}), 6.56(\mathrm{~s}, 1 \mathrm{H}), 7.87(\mathrm{~d}, J=7.6 \mathrm{~Hz}, 2 \mathrm{H})$; HRMS (FAB) (M) calcd for $\mathrm{C}_{17} \mathrm{H}_{24} \mathrm{Si} 256.1674$, found 256.1654 .

(3-Butyloct-3E-en-1-yn-1-yl)trimethylsilane (4i): $69 \%$ (E/Z = 1/1.5); characteristic ${ }^{1} \mathrm{H}$ NMR signals for $E$ isomer $\delta 0.19(\mathrm{~s}, 9 \mathrm{H})$, $5.92(\mathrm{t}, J=7.6 \mathrm{~Hz}, 1 \mathrm{H}) ; Z$ isomer ${ }^{1} \mathrm{H} \mathrm{NMR}\left(\mathrm{CDCl}_{3}, 400 \mathrm{MHz}\right)$ $\delta 0.20(\mathrm{~s}, 9 \mathrm{H}), 0.87-0.95(\mathrm{~m}, 6 \mathrm{H}), 1.26-1.42(\mathrm{~m}, 6 \mathrm{H}), 1.44-1.53$ $(\mathrm{m}, 2 \mathrm{H}), 2.05-2.14(\mathrm{~m}, 2 \mathrm{H}), 2.26(\mathrm{q}, J=7.2 \mathrm{~Hz}, 2 \mathrm{H}), 5.69(\mathrm{t}$, $J=7.2 \mathrm{~Hz}, 1 \mathrm{H}$ ); HRMS (FAB) (M) calcd for $\mathrm{C}_{15} \mathrm{H}_{28} \mathrm{Si} 236.1960$, found 236.1961.

[3-Butyl-4-(4-trifluomethylphenyl)but-3E-en-1-yn-1-yl]trimethylsilane $(\mathbf{4 j}): 48 \%(E / Z=3.3 / 1) ; E$ isomer ${ }^{1} \mathrm{H} \mathrm{NMR}\left(\mathrm{CDCl}_{3}, 400\right.$ MHz) $\delta 0.26(\mathrm{~s}, 9 \mathrm{H}), 0.93(\mathrm{t}, J=7.2 \mathrm{~Hz}, 3 \mathrm{H}), 1.32-1.46(\mathrm{~m}, 2$ H), 1.59-1.70 (m, $2 \mathrm{H}), 2.37$ (t, $J=7.8 \mathrm{~Hz}, 2 \mathrm{H}), 6.90$ (s, $1 \mathrm{H})$, $7.34(\mathrm{~d}, J=8.2 \mathrm{~Hz}, 2 \mathrm{H}), 7.59(\mathrm{~d}, J=8.2 \mathrm{~Hz}, 2 \mathrm{H})$; characteristic ${ }^{1} \mathrm{H}$ NMR signals for $Z$ isomer $\delta 0.28(\mathrm{~s}, 9 \mathrm{H}), 0.97(\mathrm{t}, J=7.4 \mathrm{~Hz}$, $3 \mathrm{H}), 6.56$ (s, $1 \mathrm{H}), 7.95$ (d, $J=8.4 \mathrm{~Hz}, 2 \mathrm{H})$; HRMS (FAB) (M) calcd for $\mathrm{C}_{18} \mathrm{H}_{23} \mathrm{~F}_{3} \mathrm{Si}$ 324.1521, found 324.1525.
[3-Butyl-4-(4-cyanophenyl)but-3E-en-1-yn-1-yl]trimethylsilane (4k): $39 \%(E / Z=1.4 / 1)$. When $0.25 \mathrm{~mL}$ of $\mathrm{Bu}_{3} \mathrm{P}$ was used in place of $\mathrm{Ph}_{3} \mathrm{P}, 89 \%$ yield of $\mathbf{4 k}$ was isolated $(E / Z=2.0 / 1)$. Under these conditions, 3ka was converted into $Z-\mathbf{4 k}$ in $71 \%$ yield and $\mathbf{3 k b}$ (94\% de) into $E-\mathbf{4 k}$ (containing $3 \% \mathrm{Z}-\mathbf{4 k}$ ) in $71 \%$ yield. $E-\mathbf{4 k}:{ }^{1} \mathrm{H}$ $\operatorname{NMR}\left(\mathrm{CDCl}_{3}, 400 \mathrm{MHz}\right) \delta 0.25(\mathrm{~s}, 9 \mathrm{H}), 0.91(\mathrm{t}, J=7.4 \mathrm{~Hz}, 3$ H), $1.30-1.42(\mathrm{~m}, 2 \mathrm{H}), 1.58-1.66(\mathrm{~m}, 2 \mathrm{H}), 2.34(\mathrm{t}, J=7.6 \mathrm{~Hz}$, $2 \mathrm{H}), 6.84(\mathrm{~s}, 1 \mathrm{H}), 7.31(\mathrm{~d}, J=8.4 \mathrm{~Hz}, 2 \mathrm{H}), 7.61(\mathrm{~d}, J=8.4 \mathrm{~Hz}$, $2 \mathrm{H}) . \mathrm{Z}-4 \mathrm{k}:{ }^{1} \mathrm{H} \mathrm{NMR}\left(\mathrm{CDCl}_{3}, 400 \mathrm{MHz}\right) \delta 0.26(\mathrm{~s}, 9 \mathrm{H}), 0.95(\mathrm{t}$, $J=7.2 \mathrm{~Hz}, 3 \mathrm{H}), 1.37-1.42(\mathrm{~m}, 2 \mathrm{H}), 1.58-1.66(\mathrm{~m}, 2 \mathrm{H}), 2.33$ (t, $J=7.6 \mathrm{~Hz}, 2 \mathrm{H}), 6.53(\mathrm{~s}, 1 \mathrm{H}), 7.58$ (d, $J=8.4 \mathrm{~Hz}, 2 \mathrm{H}), 7.92$ (d, $J=8.4 \mathrm{~Hz}, 2 \mathrm{H}$ ); HRMS (FAB) (M) calcd for $\mathrm{C}_{18} \mathrm{H}_{24} \mathrm{NSi}$ 282.1678 , found 282.1678 .

[3-Butyl-4-(4-tolyl)but-3E-en-1-yn-1-yl]trimethylsilane (4I): 67\% $(E / Z=2.1 / 1) ; E$ isomer ${ }^{1} \mathrm{H} \mathrm{NMR}\left(\mathrm{CDCl}_{3}, 400 \mathrm{MHz}\right) \delta 0.25(\mathrm{~s}, 9$ $\mathrm{H}), 0.86(\mathrm{t}, J=7.4 \mathrm{~Hz}, 3 \mathrm{H}), 1.25-1.35(\mathrm{~m}, 2 \mathrm{H}), 1.52-1.67(\mathrm{~m}$, $2 \mathrm{H}), 2.22(\mathrm{t}, J=7.6 \mathrm{~Hz}, 2 \mathrm{H}), 2.27(\mathrm{~s}, 3 \mathrm{H}), 6.90(\mathrm{~s}, 1 \mathrm{H})$, 7.09-7.24 (m, $4 \mathrm{H})$; characteristic ${ }^{1} \mathrm{H}$ NMR signals for $Z$ isomer $\delta{ }^{1} \mathrm{H} \mathrm{NMR}\left(\mathrm{CDCl}_{3}, 400 \mathrm{MHz}\right) \delta 0.19(\mathrm{~s}, 9 \mathrm{H}), 0.96(\mathrm{t}, J=7.2 \mathrm{~Hz}$, $3 \mathrm{H}), 1.19(\mathrm{~d}, J=6.4 \mathrm{~Hz}, 6 \mathrm{H}), 1.35-1.46(\mathrm{~m}, 2 \mathrm{H}), 2.30-2.36$ (m, $5 \mathrm{H}), 6.70$ (s, $1 \mathrm{H}), 8.09-8.14(\mathrm{~m}, 1 \mathrm{H})$; HRMS (FAB) $\left(\mathrm{M}^{+}\right)$ calcd for $\mathrm{C}_{18} \mathrm{H}_{26} \mathrm{Si} 270.1804$, found 270. 1801.

2-Butyl-1-(4-tert-butoxycarbonylphenyl)oct-1E-en-3-yne (4m): $62 \%(E / Z=1.6 / 1) ; E$ isomer ${ }^{1} \mathrm{H}$ NMR $\left(\mathrm{CDCl}_{3}, 400 \mathrm{MHz}\right) \delta$ 0.88-0.99 (m, $6 \mathrm{H}), 1.29-1.66(\mathrm{~m}, 17 \mathrm{H}$, embodied a singlet at 1.60 for tert-butyl group), $2.28-2.40(\mathrm{~m}, 2 \mathrm{H}), 2.45(\mathrm{t}, J=7.2$ $\mathrm{Hz}, 2 \mathrm{H}), 6.76(\mathrm{~s}, 1 \mathrm{H}), 7.26(\mathrm{~d}, J=8.0 \mathrm{~Hz}, 2 \mathrm{H}), 7.93(\mathrm{~d}, J=8.0$ $\mathrm{Hz}, 2 \mathrm{H})$; characteristic ${ }^{1} \mathrm{H}$ NMR signals for $Z$ isomer $\delta{ }^{1} \mathrm{H}$ NMR $\left(\mathrm{CDCl}_{3}, 400 \mathrm{MHz}\right) \delta 6.48(\mathrm{~s}, 1 \mathrm{H}), 7.83(\mathrm{~d}, J=8.4 \mathrm{~Hz}, 2 \mathrm{H}), 7.91$ (d, $J=8.4 \mathrm{~Hz}, 2 \mathrm{H}$ ); HRMS (FAB) (M) calcd for $\mathrm{C}_{23} \mathrm{H}_{32} \mathrm{O}_{2}$ 340.2402 , found 340.2398 .

3-Butyl-2-methylnon-2-en-4-yne (4n): $17 \%\left(\mathrm{Bu}_{3} \mathrm{P}\right.$ was used); ${ }^{1} \mathrm{H}$ NMR $\left(\mathrm{CDCl}_{3}, 400 \mathrm{MHz}\right) \delta 0.92(\mathrm{t}, J=7.2 \mathrm{~Hz}, 3 \mathrm{H}), 0.94$ (t, $J=$ $7.2 \mathrm{~Hz}, 3 \mathrm{H}), 1.26-1.38(\mathrm{~m}, 4 \mathrm{H}), 1.41-1.57$ (m, $4 \mathrm{H}), 1.73$ (s, 3 H), 1.93 (s, $3 \mathrm{H}), 2.12$ (t, $J=7.6 \mathrm{~Hz}, 2 \mathrm{H}), 2.36$ (t, $J=6.8 \mathrm{~Hz}, 2$ $\mathrm{H}) ;{ }^{13} \mathrm{C} \mathrm{NMR}\left(100 \mathrm{MHz}, \mathrm{CDCl}_{3}\right) \delta 13.8,14.2,19.3,19.7,22.1$, 22.5, 23.7, 31.0, 31.4, 32.2, 81.3, 91.8, 117.6, 137.3; HRMS $\left(\mathrm{EI}^{+}\right)$ (M) calcd for $\mathrm{C}_{14} \mathrm{H}_{24}$ 192.1878, found 192.1873.

2-Butyl-1,1-diphenyloct-1-en-3-yne (40): $28 \%\left(\mathrm{Bu}_{3} \mathrm{P}\right.$ was used); ${ }^{1} \mathrm{H} \mathrm{NMR}\left(\mathrm{CDCl}_{3}, 400 \mathrm{MHz}\right) \delta 0.85(\mathrm{t}, J=7.6 \mathrm{~Hz}, 3 \mathrm{H}), 0.88(\mathrm{t}$, $J=7.4 \mathrm{~Hz}, 3 \mathrm{H}), 1.22-1.38(\mathrm{~m}, 4 \mathrm{H}), 1.39-1.48(\mathrm{~m}, 2 \mathrm{H})$, $1.55-1.65(\mathrm{~m}, 2 \mathrm{H}), 2.21(\mathrm{t}, J=7.4 \mathrm{~Hz}, 2 \mathrm{H}), 2.28(\mathrm{t}, J=6.8 \mathrm{~Hz}$, $2 \mathrm{H}), 7.11-7.42(\mathrm{~m}, 10 \mathrm{H}) ;{ }^{13} \mathrm{C} \mathrm{NMR}\left(100 \mathrm{MHz}, \mathrm{CDCl}_{3}\right) \delta 14.1$, $14.4,19.7,22.2,22.6,30.9,31.5,34.7,81.5,94.0,122.0,126.4$, $126.5,127.0,127.6,129.2,129.4,141.3,141.9,145.0$; HRMS (FAB) (M) calcd for $\mathrm{C}_{24} \mathrm{H}_{28} 316.2191$, found 316.2193.

(5-Phenyl-4-isopropyl-pent-4E-en-2-yn-1-yl tert-butyldimethylsilyl ether (4p): $38 \%(E / Z=1.6 / 1) ; E$ isomer ${ }^{1} \mathrm{H}$ NMR $\left(\mathrm{CDCl}_{3}\right.$, $400 \mathrm{MHz}) \delta 0.19(\mathrm{~s}, 9 \mathrm{H}), 0.96(\mathrm{~s}, 6 \mathrm{H}), 2.14(\mathrm{~d}, J=6.8 \mathrm{~Hz}, 6 \mathrm{H})$, 3.06 (sept, $J=6.8 \mathrm{~Hz}, 1 \mathrm{H}$ ), 4.53 (s, $2 \mathrm{H}$ ), 6.77 (s, $1 \mathrm{H}$ ), $7.21-7.39$ (m, $5 \mathrm{H}$ ); characteristic ${ }^{1} \mathrm{H}$ NMR signals for $Z$ isomer $\delta 0.17$ (s, 9 H), 0.96 (s, $6 \mathrm{H}), 2.19$ (d, $J=6.8 \mathrm{~Hz}, 6 \mathrm{H}), 2.58$ (sept, $J=6.7$ $\mathrm{Hz}, 1 \mathrm{H}), 4.58$ (s, $2 \mathrm{H}), 6.56$ (s, $1 \mathrm{H}), 7.78-7.84$ (m, $2 \mathrm{H})$; HRMS $\left(\mathrm{EI}^{+}\right)(\mathrm{M})$ calcd for $\mathrm{C}_{20} \mathrm{H}_{30} \mathrm{OSi}$ 314.2066, found 314.2062.

1-(Naphthyl)-2-phenyl-2,2-bis(propylthio)ethanol (8). Under $\mathrm{N}_{2}$ atmosphere, to a THF solution $(50 \mathrm{~mL})$ of benzaldehyde bispropyldithioacetal $(1.20 \mathrm{~g}, 5 \mathrm{mmol})$ cooled at $-78{ }^{\circ} \mathrm{C}$ was added dropwise LDA (2.5 mL, 1.0 equiv, 2.0 M THF/n-heptane/ethylbenzene solution). After the solution was stirred for $2 \mathrm{~h}$, 1-naphthaldehyde $(0.68 \mathrm{~mL}, 5.0 \mathrm{mmol})$ in THF $(10 \mathrm{~mL})$ was added. The mixture was gradually warmed to rt and stirred for $8 \mathrm{~h}$, quenched with sat. $\mathrm{NH}_{4} \mathrm{Cl}$, washed with brine, and extracted with ether. The organic layer was dried $\left(\mathrm{MgSO}_{4}\right)$ and filtered and the filtrate was evaporated in vacuo to give the residue, which was chromatographed on silica gel (hexane/EtOAc $=10 / 1)$ to give 8 as a light yellow solid $(0.89 \mathrm{~g}, 45 \%)$ : mp $78-79{ }^{\circ} \mathrm{C} ;{ }^{1} \mathrm{H} \mathrm{NMR}\left(\mathrm{CDCl}_{3}, 400\right.$ $\mathrm{MHz}) \delta 0.90(\mathrm{t}, J=7.2 \mathrm{~Hz}, 3 \mathrm{H}), 1.16(\mathrm{t}, J=7.4 \mathrm{~Hz}, 3 \mathrm{H})$, 1.45-1.55 (m, $2 \mathrm{H}), 1.73-1.87(\mathrm{~m}, 2 \mathrm{H}), 2.23-2.31(\mathrm{~m}, 1 \mathrm{H})$, 2.47-2.55 (m, 1 H), 2.89-3.07 (m, $2 \mathrm{H}), 3.88$ (s, $1 \mathrm{H}), 5.84$ (s, 1 
H), $6.68(\mathrm{~d}, J=7.2 \mathrm{~Hz}, 1 \mathrm{H}), 7.14-7.29(\mathrm{~m}, 4 \mathrm{H}), 7.40-7.46(\mathrm{~m}$, $2 \mathrm{H}), 7.47-7.53(\mathrm{~m}, 2 \mathrm{H}), 7.73(\mathrm{~d}, J=8.0 \mathrm{~Hz}, 1 \mathrm{H}), 7.78-7.84$ $(\mathrm{m}, 1 \mathrm{H}), 8.08-8.14(\mathrm{~m}, 1 \mathrm{H}) ;{ }^{13} \mathrm{C}$ NMR $\left(\mathrm{CDCl}_{3}, 100 \mathrm{MHz}\right) \delta$ $14.1,14.3,22.0,22.4,32.0,33.1,72.5,123.7,124.2,124.8,125.1$, 127.3, 127.6, 128.3, 128.4, 130.1, 132.1, 132.9, 133.1, 136.7; IR $(\mathrm{KBr}) \quad v 3449 \mathrm{~cm}^{-1}$; HRMS (ESI) $(\mathrm{M}+\mathrm{Na})^{+}$calcd for $\mathrm{C}_{24} \mathrm{H}_{28} \mathrm{OS}_{2} \mathrm{Na} 419.1479$, found 419.1489 .

1-(2-Phenyl-1,2-bis(propylthio)vinyl)naphthalene (11). In a manner similar to that described in the general procedure for the preparation 4, 8 (99 $\mathrm{mg}, 0.25 \mathrm{mmol})$ and $\mathrm{PBu}_{3}(0.25 \mathrm{~mL}, 1 \mathrm{mmol})$ were converted to 11 as a colorless liquid $(65 \mathrm{mg}, 69 \%, E / Z=$ 1/2): characteristic ${ }^{1} \mathrm{H}$ NMR signals for $E$ isomer $\delta 0.64(\mathrm{t}, J=$ $7.4 \mathrm{~Hz}, 3 \mathrm{H}), 0.68(\mathrm{t}, J=7.4 \mathrm{~Hz}, 3 \mathrm{H}), 1.22-1.37(\mathrm{~m}, 4 \mathrm{H}) ; Z$ isomer ${ }^{1} \mathrm{H}$ NMR $\left(\mathrm{CDCl}_{3}, 400 \mathrm{MHz}\right) \delta 0.79(\mathrm{t}, J=7.4 \mathrm{~Hz}, 3 \mathrm{H})$, $0.99(\mathrm{t}, J=7.4 \mathrm{~Hz}, 3 \mathrm{H}), 1.37-1.46(\mathrm{~m}, 2 \mathrm{H}), 1.59-1.66(\mathrm{~m}, 2$ H), 2.05-2.14 (m, $2 \mathrm{H}), 2.45(\mathrm{t}, J=7.2 \mathrm{~Hz}, 2 \mathrm{H}), 6.93-6.97(\mathrm{~m}$, $2 \mathrm{H}), 7.07-7.09(\mathrm{~m}, 2 \mathrm{H}), 7.17(\mathrm{~d}, J=8.0 \mathrm{~Hz}, 1 \mathrm{H}), 7.24-7.30$ (m, 1 H), 7.41-7.60 (m, 1 H), 7.60-7.67 (m, $2 \mathrm{H}), 7.78(\mathrm{~d}, J=$ $8.0 \mathrm{~Hz}, 1 \mathrm{H}), 7.89-7.95$ (m, $1 \mathrm{H}), 8.21-8.25$ (m, $1 \mathrm{H})$; HRMSFAB $\left(\mathrm{M}^{+}\right)$calcd for $\mathrm{C}_{24} \mathrm{H}_{26} \mathrm{~S}_{2} 378.1476$, found 378.1480.

1,4-Bis(2-butyloct-1E-en-3-yn-1-yl)benzene (13). Under $\mathrm{N}_{2}$ atmosphere, to a THF solution $(50 \mathrm{~mL})$ of $\mathbf{1 d}^{7}(1.21 \mathrm{~g}, 5.0 \mathrm{mmol}$, 1.00 equiv) cooled at $-78^{\circ} \mathrm{C}$ was added dropwise BuLi $(2.2 \mathrm{~mL}$, 1.10 equiv, $2.5 \mathrm{M}$ hexane solution). After the solution was stirred for $1 \mathrm{~h}$, terephthaldehyde $(0.34 \mathrm{~g}, 2.5 \mathrm{mmol}, 0.50$ equiv) in THF $(20 \mathrm{~mL})$ was added dropwise. The mixture was gradually warmed to $\mathrm{rt}$ and stirred for $8 \mathrm{~h}$, quenched with sat. $\mathrm{NH}_{4} \mathrm{Cl}$, washed with brine, and extracted with ether. The organic layer was dried $\left(\mathrm{MgSO}_{4}\right)$ and filtered and the filtrate was evaporated in vacuo to give the residue, which was chromatographed on silica gel (hexane/ EtOAc $=10 / 1)$ to give corresponding diol (1.26 g, 69\%).

A THF solution $(5 \mathrm{~mL})$ of the diol $(368 \mathrm{mg})$ and $\mathrm{PPh}_{3}(524 \mathrm{mg}$, $2 \mathrm{mmol}, 4.00$ equiv) was allowed to react with DIAD $(0.40 \mathrm{~mL}, 2$ mmol, 4.00 equiv) and the mixture was stirred at $\mathrm{rt}$ for $18 \mathrm{~h}$, then evaporated in vacuo to give the residue, which was chromatographed on silica gel (hexane) to give $\mathbf{1 3}$ (78 mg, 39\%, 27\% in two steps, $E E / E Z=1 / 3.2)$ : characteristic ${ }^{1} \mathrm{H}$ NMR signals for $E E$ isomer $\delta 6.74(\mathrm{~s}, 2 \mathrm{H}), 7.20(\mathrm{~s}, 4 \mathrm{H}) ; E Z$ isomer $\delta 0.90-0.99(\mathrm{~m}$, $12 \mathrm{H}), 1.32-1.67(\mathrm{~m}, 16 \mathrm{H}), 2.29(\mathrm{t}, J=7.2 \mathrm{~Hz}, 2 \mathrm{H}), 2.39(\mathrm{t}, J$ $=6.8 \mathrm{~Hz}, 4 \mathrm{H}), 2.44-2.50(\mathrm{~m}, 2 \mathrm{H}), 6.43(\mathrm{~s}, 1 \mathrm{H}), 6.73(\mathrm{~s}, 1 \mathrm{H})$, $7.19(\mathrm{~d}, J=8.4 \mathrm{~Hz}, 2 \mathrm{H}), 7.78(\mathrm{~d}, J=8.4 \mathrm{~Hz}, 2 \mathrm{H})$; HRMS (FAB) (M) calcd for $\mathrm{C}_{30} \mathrm{H}_{42}$ 402.3287, found 402.3292 .

2-Isopropyl-2-(2-formylethynyl)-1,3-dithiolane (14). To a DCM solution $(100 \mathrm{~mL})$ of $1 \mathrm{e}(2.02 \mathrm{~g}, 10 \mathrm{mmol})$ was added PCC $(2.16$ $\mathrm{g}, 10 \mathrm{mmol})$ and celite $(2.16 \mathrm{~g})$ at rt. After being stirred for $12 \mathrm{~h}$, the mixture was filtered and the filtrate was evaporated in vacuo to give the residue, which was chromatographed on silica gel (hexane/ $\mathrm{CHCl}_{3}=3 / 1$ ) to give $\mathbf{1 4}$ as an orange yellow liquid (1.74 g, 87\%). ${ }^{1} \mathrm{H} \mathrm{NMR}\left(\mathrm{CDCl}_{3}, 400 \mathrm{MHz}\right) \delta 1.24(\mathrm{~d}, J=6.7 \mathrm{~Hz}, 6 \mathrm{H}), 2.34$ (sept, $J=6.7 \mathrm{~Hz}, 1 \mathrm{H}), 3.40-3.58(\mathrm{~m}, 4 \mathrm{H}), 9.28(\mathrm{~s}, 1 \mathrm{H}) ;{ }^{13} \mathrm{C}$ NMR (100 MHz, $\left.\mathrm{CDCl}_{3}\right) \delta 21.2,39.9,40.0,65.3,84.2,97.2,176.4$; IR (KBr) $v 1662 \mathrm{~cm}^{-1}$; HRMS (FAB) $(\mathrm{M}+\mathrm{H})$ calcd for $\mathrm{C}_{9} \mathrm{H}_{13} \mathrm{OS}_{2}$ 201.0408, found 201.0410.

Alcohol 15. According to general procedure for the preparation of $\mathbf{3}, \mathbf{1 c}^{7}$ (1.29 g, $\left.5.0 \mathrm{mmol}\right)$ and $\mathbf{1 4}(1.00 \mathrm{~g}, 5.0 \mathrm{mmol})$ were converted to $\mathbf{1 5}$ as a pale yellow liquid $(1.16 \mathrm{~g}, 45 \%$, $\mathrm{dr}$ ratio $=$ 1.3/1). Major diastereomer ${ }^{1} \mathrm{H} \mathrm{NMR}\left(\mathrm{CDCl}_{3}, 400 \mathrm{MHz}\right) \delta 0.20(\mathrm{~s}$, $9 \mathrm{H}), 0.88-0.96(\mathrm{~m}, 6 \mathrm{H}), 1.24(\mathrm{~d}, J=6.8 \mathrm{~Hz}, 6 \mathrm{H}), 1.30-1.47$ (m, $4 \mathrm{H}), 1.49-1.73(\mathrm{~m}, 4 \mathrm{H}), 2.23-2.32(\mathrm{~m}, 1 \mathrm{H}), 2.56(\mathrm{t}, J=$ $6.6 \mathrm{~Hz}, 2 \mathrm{H}), 2.68-3.16(\mathrm{~m}, 5 \mathrm{H}), 3.35-3.42$ (m, $2 \mathrm{H}), 3.48-3.57$ $(\mathrm{m}, 2 \mathrm{H}), 4.44(\mathrm{~d}, J=6.4 \mathrm{~Hz}, 1 \mathrm{H})$; characteristic ${ }^{1} \mathrm{H}$ NMR signals for the minor diastereomer $\delta 0.21(\mathrm{~s}, 9 \mathrm{H}), 2.57(\mathrm{t}, J=6.6 \mathrm{~Hz}, 2$ $\mathrm{H}), 4.41(\mathrm{~d}, J=9.6 \mathrm{~Hz}, 1 \mathrm{H})$; IR $(\mathrm{KBr}) v 3421 \mathrm{~cm}^{-1}$; HRMS (FAB) $(\mathrm{M}+\mathrm{H})$ calcd for $\mathrm{C}_{25} \mathrm{H}_{45} \mathrm{OSiS}_{4}$ 517.2123, found 517.2128.

Endiyne 16. According to teh general procedure for the preparation of 4, 15 (258 mg, $0.5 \mathrm{mmol}$ ) and $\mathrm{PPh}_{3}$ were converted to 16 as a colorless liquid $(119 \mathrm{mg}, 68 \%, E / Z=1 / 1.4)$ : characteristic ${ }^{1} \mathrm{H}$ NMR signals for $E$ isomer $\delta 0.20(\mathrm{~s}, 9 \mathrm{H}), 0.93(\mathrm{t}, J=7.4 \mathrm{~Hz}, 3$ H), 1.22 (d, $J=6.8 \mathrm{~Hz}, 6 \mathrm{H}), 2.31$ (sept, $J=6.8 \mathrm{~Hz}, 1 \mathrm{H}$ ), 2.36 (t, $J=7.4 \mathrm{~Hz}, 2 \mathrm{H}), 5.86(\mathrm{~s}, 1 \mathrm{H}) ; Z$ isomer $\delta 0.21(\mathrm{~s}, 9 \mathrm{H}), 0.91$ $(\mathrm{t}, J=7.2 \mathrm{~Hz}, 3 \mathrm{H}), 1.28(\mathrm{~d}, J=6.6 \mathrm{~Hz}, 6 \mathrm{H}), 1.29-1.41(\mathrm{~m}$, $2 \mathrm{H}), 1.46-1.58(\mathrm{~m}, 2 \mathrm{H}), 2.15-2.21(\mathrm{~m}, 2 \mathrm{H}), 2.32$ (sept, $J=6.6$ $\mathrm{Hz}, 1 \mathrm{H}), 3.36-3.46(\mathrm{~m}, 2 \mathrm{H}), 3.46-3.60(\mathrm{~m}, 2 \mathrm{H}), 5.71(\mathrm{t}, J=1.2$ $\mathrm{Hz}, 1 \mathrm{H})$; HRMS (FAB) $(\mathrm{M}+\mathrm{H})$ calcd for $\mathrm{C}_{19} \mathrm{H}_{31} \mathrm{SiS}_{2} 351.1636$, found 351.1631 .

Acknowledgement. We thank the National Science Council and the National Taiwan University of the Republic of China for financial support.

Supporting Information Available: ${ }^{1} \mathrm{H}$ NMR spectra of $1 \mathbf{1 e}$, 3a-f, 3h-p, 3ka, 3kb, 4a-p, Z-4k, $E-4 \mathbf{k}, 8,11$, and 13-16. This material is available free of charge via the Internet at http://pubs.acs.org.

JO8013885 